\title{
ANOMALÍAS Y MEJORAS EN LAS ESTADÍSTICAS DE MIGRACIÓN INTERNACIONAL DURANTE LAS ÚLTIMAS DÉCADAS
}

\section{IMPROVEMENTS AND ANOMALIES IN INTERNATIONAL MIGRATION STATISTICS DURING THE LAST DECADES}

\author{
IGNACIO DUQUE ignacio.duque.rodriguez@ine.es \\ Instituto Nacional de Estadística. España
}

\begin{abstract}
RESUMEN
¿Por qué se ha avanzado poco, o incluso se ha retrocedido, en la disponibilidad de estadísticas de migración internacional con una aceptable cobertura y calidad en el último siglo? En este artículo se hace un repaso de los principales diagnósticos internacionales y se discuten distintas hipótesis explicativas de esta anomalía en la estadística oficial, detallando como ejemplo los flujos interiores a la Unión Europea y revisando la situación española. Por otra parte, se mencionan las iniciativas más relevantes de mejora, así como las consecuencias de todo este panorama en relación con el debate democrático sobre los flujos y las políticas migratorias a nivel internacional.
\end{abstract}

\section{Palabras clave}

Comparabilidad, Etnicidad, Extranjeros, Migrantes, Población.

\begin{abstract}
Why has international migration statistics had short improvements during the last century? Why are they partially worsening? The text analyzes the main international reports on quality of international flux and tests the more relevant hypothesis about this intriguing anomaly in the landscape of Official Statistics. A detailed review is made about migration flux inside European Union and the situation of Spain in this field. The paper presents a deep review of the best initiatives and practices for improving the knowledge of human flux associated to globalization and the relevance of accurate statistics for any democratic debate about migration trends and migration policies.
\end{abstract}

\section{KEYWORDS}

Comparability, Ethnicity, Foreigners, Migrants, Population. 


\section{INTRODUCCIÓN}

Después del horror producido por las devastaciones desarrolladas durante la Segunda Guerra Mundial y antes de que se iniciasen otro ciclo de conflictos al socaire de los nuevos procesos de acumulación, la UNESCO tomó una serie de iniciativas educativas y culturales ${ }^{1}$, tratando de responder a la pregunta de por qué se había producido tanta barbarie en el hogar del viejo mundo que ha gustado de verse a sí mismo como alumbrador de las más nobles ideas de igualdad, fraternidad y progreso. Siendo de general conocimiento que la cristalización de determinados conceptos (como la raza, la cultura, la civilización) habían representado un papel clave, se encargó a Claude Levi-Strauss un informe que permitiese evaluar la relación entre los diversos grupos y el género humano y atisbar una estrategia que permitiese dificultar la cristalización de derivas identitarias que representaron un papel clave en los conflictos territoriales, civiles y militares.

Partiendo (en su texto de 1952 Raza e historia) de la constatación empírica de que quizá la mayor invariante histórica es que no hay ninguna cultura que considere que los no pertenecientes a ella tienen todos los atributos de un ser humano completo $^{2}$ y una vez desenganchados los vagones del etnocentrismo y el pseudo-evolucionismo cultural, Levi-Strauss llega a formular la paradoja final de las condiciones de progreso de y entre las culturas (con una cierta resonancia a segunda ley de la termodinámica): cada cultura tiende a negar lo distinto, pero la innovación (que explica el éxito como especie) sólo se produce por el contacto con lo diferente. Dos décadas después (Lévi-Strauss 2001 [1971], 164-166) el gran antropólogo refuerza sus reflexiones remarcando la conexión del fenómeno del racismo, la xenofobia o el ultranacionalismo, no con evoluciones intelectuales 0 de escuelas de pensamiento, sino con las condiciones de poder y acceso

\footnotetext{
${ }^{1}$ Ignacio Duque trabaja en el Instituto Nacional de Estadística y, como es usual y conveniente señalar, las opiniones reflejadas en el texto no tienen por qué coincidir con las del INE en tanto que institución. La redacción fundamental de éste texto se terminó entre diciembre de 2009 y febrero de 2010. Se agradecen mucho las sugerencias y comentarios del/de la evaluardor/a que han permitido precisar el contenido y ganar en claridad expositiva. La mayoría de ellas aunaban buena intención y dificultades prácticas, como el caso de la Historia de la Humanidad no centrada en los países desarrollados (History of the Scientific and Cultural Development of Mankind, iniciada en 1946 con ayuda de una comisión cientifica presidida por Charles Morazé, http://www.unesco.org/culture/humanity/)

${ }^{2}$ «On sait, en effet, que la notion d'humanité, englobant, sans distinction de race ou de civilisation, toutes les formes de l'espèce humaine, est d'apparition fort tardive et d'expansion limitée ... pour de vastes fractions de l'espèce humaine et pendant des dizaines de millénaires, cette notion paraît être totalement absente. L'humanité cesse aux frontières de la tribu, du groupe linguistique, parfois même du village; à tel point qu'un grand nombre de populations dites primitives se désignent d'un nom qui signifie «hommes» (ou parfois -dirons-nous avec plus de discrétion- les «bons», les «excellents», les «complets»), impliquant ainsi que les autres tribus, groupes ou villages ne participent pas des vertus - ou même de la nature - humaines, mais son tout au plus composés de «mauvais», de "méchants», de "singes de terre» ou d'«œufs de pou»» (Lévi-Strauss 2001 [1942], 45)
} 
desigual a los recursos y en el contexto de sobrepoblación relativa e impacto en los ecosistemas naturales.

No se pueden entender las peculiaridades de las estadísticas de migración internacional sin reconocer su relación con los aspectos de identidad y diversidad cultural, con el debate sobre el carácter nacional ${ }^{3}$ y otras categorías de auto-representación de los grupos humanos. Es lo que se describirá como la peculiar "anomalía" de este campo especial de la información estadística.

Habitualmente se considera que la actividad de producción de información estadística es en nuestras avanzadas sociedades un cometido altamente tecnificado, que aplica conceptos obvios y métodos científicos contrastados, para obtener resultados para todos los usos y usuarios. Sin embargo, si tomamos al pie de la letra tales afirmaciones, que en realidad no son sino un loable deseo de objetividad bien difícil de alcanzar en la práctica, el viaje por las estadísticas de migraciones resulta sumamente errático.

Por suerte toda una serie de trabajos y reflexiones nos permiten hoy trazar la genealogía de la actividad estadística como una especie de confluencia de tres grandes mundos: a) matemáticas aplicadas, b) meliorismo económico y social y c) producción estatal de información. Esto ha sido bien estudiado en relación con aquellos aspectos de la disciplina que aparecen más claramente como un producto de la "pura razón", cual es el caso de los trabajos sobre el análisis de la probabilidad 0 al estudio de la herencia (el envés del concepto de raza) por el triángulo Galton-Edgeworth-Yule (Stigler, 1986 y 1989; Gigerentzer et al., 1989), así como a la peculiar historia de alguna de las herramientas descriptivas por antonomasia de los fenómenos demográficos, como es la Tabla de Mortalidad (Bras 2000). Otros analistas han puesto más énfasis en la particular imbricación entre producción estatal de información y conflictos históricos y sociales en distintos contextos (Desrosières 1993; Tooze 2001) o en la peculiar complejidad que requiere la búsqueda del consenso social sobre la fiabilidad de los números (Porter, 1995). No han faltado análisis de operaciones concretas, como el caso del Censo americano (Anderson 1988) o las aportaciones españolas (Arribas y Barbut 2002; AHEPE 2002, 2004 y 2006). En consonancia con su encumbrado papel en el pensamiento contemporáneo los métodos econométricos han sido objeto de profundos análisis, hoy tan relevantes, entre los que los que destacaría como especialmente iluminadores los de Israel (1986; Ingrao e Israel 2000), Walter y Brian (2007) y Brian (2009).

Otro comentario necesario se refiere a la distinción interior / internacional en relación con las migraciones, un asunto tan obvio como frecuentemente olvidado. En la medida en que son las fronteras estatales las que determinan cuándo un cambio de residencia se convierte en una migración internacional, el recuento de la misma está extraordina-

\footnotetext{
${ }^{3}$ Como ha sido rastreado en nuestro contexto por el mejor representante de la misma tradición etnológica, en un texto al que los años no le restan la recomendabilidad (Caro, 2004 [1970]) y que fue precisamente redactado por el estupor que produjo a Julio Caro la moda de los años 60 de la antropología americana relativa a los national studies.
} 
riamente afectado por las características de esta malla y especialmente por el tamaño de las unidades que la forman. Pequeños desplazamientos, en términos físicos, pueden ser cualificados de internacionales (piénsese en la movilidad en el entorno de Luxemburgo) y enormes viajes (dentro de los grandes países-continente como Rusia, Brasil - EEUU) son migraciones internas. Además si el recuento de migrantes se realiza a través del stock de nacidos en el extranjero ${ }^{4}$, añadimos a dicha cuenta muchos millones de personas que jamás han traspasado una frontera, pero que debido al cambio de los límites de los países se han convertido en nacidos en un país distinto, siendo lo contrario igualmente cierto.

Además, más allá de la distancia física podemos hablar de las distancias sociales que las personas recorren. Sin duda alguna muchos de los problemas asociados a las migraciones (y las demandas de información que se plantean) están determinados por esta distancia social que se suele asociar a la migración internacional, pero que no es exclusiva de la misma. Es un tema que José Moya ha tratado magistralmente al analizar los problemas de integración de los próximos en el contexto de la emigración española a Buenos Aires (Moya, 1998) y que también nos recuerda la pluma al tiempo ácida y empírica de Engels, relatando el trato sufrido por los inmigrantes irlandeses (que no cambiaban de Estado) en los suburbios de las ciudades industriales inglesas ${ }^{5}$.

\section{LA APARENTE ANOMALÍA DE LAS ESTADÍSTICAS SOBRE MIGRACIÓN INTERNACIONAL Y SUS CONSECUENCIAS}

Cualquiera que sea el ámbito que observemos de la producción estadística regular permite apreciar los enormes avances observados en las últimas décadas, máxime si nos extendemos a revisar la última centuria. Sea la demografía, la salud, las cuentas nacionales, los precios, las condiciones de vida o el uso del tiempo, en todos estos campos los avances en el conocimiento han sido manifiestos y aunque el diagnóstico sobre la extensión territorial de dichos avances ofrece más sombras, tampoco resulta despreciable. Lo mismo se podría decir no sólo de los temas sino de las perspectivas adoptadas, de la riqueza de los soportes o de la profundidad de acceso a la información. En todos los ámbitos también se desarrollan clasificaciones más detalladas y normalizadas, abarcando más dimensiones, con más detalle territorial y para conjuntos más integrados de información.

Sin embargo, el ámbito de las migraciones internacionales ha sido certeramente caracterizado como el de "una centuria de recomendaciones ignoradas" (Santo Tomás,

\footnotetext{
${ }^{4}$ Para una discusión de este impacto en las estimaciones a nivel mundial véase United Nations (2009: 4-5).

5 "... causes and results of this immigration... The rapid extension of English industry could not have taken place if England had not possessed in the numerous and impoverished population of Ireland a reserve at command" (Engels 1999 [1845]: 101).
} 
Summers y Clemens 2009: 3). Efectivamente parece que en el ámbito de las instituciones internacionales que están detrás de las mejoras estadísticas se verifica desde hace más de cien años una rutina por la cual cada nueva evaluación recuerda los limitados progresos, e incluso retrocesos, sobre la anterior, señala en qué medida han sido desatendidas las recomendaciones previas y, finalmente, intenta ensayar una estrategia más realista, que hasta el momento se ha visto abocada a un nuevo fracaso, si se revisa una década después. Los primeros jalones de esta evolución serían los esfuerzos del Instituto Internacional de Estadística en 1891 por disponer de stocks de extranjeros en cada país (Falkner 1895) o de la misma institución por distinguir entre inmigrantes permanentes o temporales (Krely y Gnanasekaran 1987). Sin duda el primer esfuerzo sistemático de estandarización de definiciones y flujos fue hecho por la Liga de Naciones a través de la Organización Internacional del Trabajo (ILO 1922), aunque finalmente, la arquitectura de estandarización fue diseñada por la serie de recomendaciones de Naciones Unidas: 1953, 1976 y las últimas de 1998.

Entre dichas recomendaciones se sitúan los diagnósticos críticos de los expertos. El más temprano sería probablemente el de Ferenczi (1925) sobre el enorme trabajo pendiente para disponer de una mínima visión completa de los flujos o del pobre avance realizado, que incluso hace una mención al enorme potencial de nuestro Archivo de Indias, pero existen varios posteriores. Entre estas revisiones críticas tiene un especial peso el diagnóstico previo de las últimas Recomendaciones de Naciones Unidas que señalan los escasos avances en cobertura u homogeneización de datos ${ }^{6}$. $Y$ los trabajos más recientes (United Nations 2004, 216) no han cambiado la impresión del panorama.

¿Cuáles son las consecuencias de esta situación? Las migraciones han constituido uno de los aspectos fundamentales en la historia de las sociedades y constituyen uno de los elementos clave de los procesos asociados a la acumulación económica desde hace varias centurias. Disponemos de información detalladísima de los intercambios de todo tipo de bienes y flujos de capitales (transferencias directas, inversiones, etc.) según sus características y destinos y según clasificaciones que proveen información bastante detallada para singulares celdillas de la Contabilidad Nacional y por el contrario no se

\footnotetext{
6 "27. The present review suggests that even in terms of mere data availability, the situation in developing countries has not improved between the late 1970s and the late 1980s, and in certain regions there are clear signs of deterioration. In addition, it is not certain that the data published by the United Nations actually conform to the definitions suggested in the 1976 recommendations. Bilsborrow and others (1997) provide some evidence suggesting that, for a number of countries, there is little consistency between the categories reported and the definitions proposed in the 1976 recommendations. Apparently, countries continued to gather data on international migration flows according to their own definitions and then made minimal adjustments to fit the data into the categories suggested by the United Nations. In many instances, however, the differences between national definitions and the 1976 recommendations are such that no amount of adjustment a posteriori would make them match" (United Nations 1998: 14). Para un resumen más actualizado del mismo diagnóstico véase el Anejo sobre la situación actual en material de recopilación de las estadísticas de migración internacional: United Nations (2004: 211-217).
} 
dispone de una panorámica general de los flujos de personas. Sin duda se trata de uno de los principales puntos ciegos del conocimiento sobre el proceso de acumulación y desarrollo económico. Estas limitaciones pueden ser vistas en varias direcciones:

a) En términos agregados, no se dispone de una panorámica completa del total de flujos de intercambios migratorios para algunas características básicas (origen, destino, nacionalidad, sexo-edad y alguna tipología significativa de motivos). Ni siquiera parece estar clara la respuesta a si el proceso de acumulación contemporánea que conocemos como globalización ${ }^{7}$ ha estimulado o no los flujos (en términos relativos a la población del planeta). Sin embargo las migraciones son la faceta humana del proceso de globalización, un compañero tan inseparable como frecuentemente denegado, sobre todo en los discursos que gustan de reducir dicho fenómeno (contra toda evidencia) a los rasgos inmateriales y tecnológicos del proceso. La mayoría de las informaciones se refieren a stocks de extranjeros y nacidos en el extranjero según países de nacimiento y/o nacionalidad (con otras características añadidas en el caso de que las fuentes sean censales), pero sin información relativamente detallada de los flujos no es posible contrastar en qué medida los diversos motores de cambio y los cambios en las políticas de gestión de los flujos están influyendo en la magnitud de los mismos.

b) Adicionalmente a la descripción de la dimensión del fenómeno, las respuestas que se esperan de la información estadística sobre migraciones internacionales requieren información relativamente detallada. Los ciudadanos, los estudiosos y las autoridades políticas se preocupan por determinados flujos y sus efectos y no consideran relevante estudiar otros (retirados o estudiantes normalmente). Por ejemplo, se busca analizar la incorporación de trabajadores a determinados sectores, supuestamente deficitarios ${ }^{8}, 0$ de unas determinadas cualificaciones, así como conocer su impacto en el mercado de trabajo, los servicios públicos, la vivienda o los efectos sobre las poblaciones autóctonas de los mismos sectores, cualificaciones o generación. Esto implica identificadores de las características de los inmigrantes recientes relativamente detalladas en todas las operaciones estadísticas regulares de un país, o disponer de dispositivos ad-hoc de captura o de combinación de fuentes. También es frecuente interrogarse sobre las causas del proceso migratorio y por qué las intervenciones de la mayoría de los Estados producen tan magros resultados cuando se trata de modificar la intensidad ${ }^{9} 0$ las características de origen o destino de flujos consolidados, o que aparezcan o desaparezcan fenómenos

\footnotetext{
${ }^{7}$ Sobre todo por lo impreciso y difuso del propio término globalización, aunque puede verse un primer acercamiento al asunto en Skaliotis y Thorogoud (2008).

${ }^{8}$ Con lo complejo que resulta esta definición, sobre todo si ha de hacerse dentro de un mecanismo regular administrativo previo a la gestión de los flujos de demanda.

${ }^{9}$ El caso más paradigmático es la migración entre Méjico y Estados Unidos a través de la frontera común.
} 
imprevistos, como nuevos colectivos o lugares de origen ${ }^{10} 0$ destino. Muchos de los ángulos ciegos son debidos al carácter asimétrico de los intereses y disponibilidades de información, siempre más centrados en las llegadas y pobres, cuando no inexistentes, en todo lo que se refiere a las emigraciones (sean salidas, retornos o movimientos encadenados o recurrentes).

c) En ocasiones la información detallada es particularmente insuficiente para dar respuesta a las demandas que se hacen a la misma, por el particular impacto de las limitaciones en cuanto al tiempo y al sujeto de observación de los movimientos migratorios internacionales. Una gran parte de los movimientos migratorios no pueden ser entendidos observando los individuos aislados, porque son las familias, las parejas o los grupos locales los que generan sus estrategias migratorias y reaccionan ante sus éxitos o fracasos relativos. Adicionalmente la información sobre la migración siempre tiene dos facetas temporales, un antes y sus pre-condiciones y un después y sus consecuencias. La recolección disjunta de las informaciones sobre el antes y el después priva a las estadísticas migratorias de una riqueza informativa que no se produce en otros ámbitos y ciertamente pocos dispositivos estadísticos están en condiciones de traspasar esta dificultad.

d) Finalmente en el estudio de las migraciones, de sus efectos y de los impactos de las medidas de todo tipo se impone la consideración del tiempo largo como el único apropiado para iluminar incluso las necesidades urgentes de toma de decisión. La perspectiva longitudinal o diacrónica de los dispositivos de información es clave, sobre todo si se trata de analizar los efectos de los procesos de integración y las paradojas de los diferentes comportamientos según grupos, países, cualificaciones, sexo o grupos sociales, analizando cómo en ocasiones durísimas condiciones alumbran el éxito y en ocasiones amplias posibilidades generan procesos de enquistamiento o de fracaso $0^{11}$. En

\footnotetext{
${ }^{10} \mathrm{Si}$ en ocasiones se ha caracterizado a la reciente inmigración española como de "inesperada" (Izquierdo, 1996), el caso de los flujos desde Rumanía, sobre todo a partir de 2005, se podrían caracterizar como lo imprevisto dentro de lo inesperado. En todo caso es todo el proceso de inmigración reciente en España en su conjunto el que requiere una explicación de síntesis (Duque 2007).

${ }^{11}$ Emmanuel Todd ha demostrado en su brillante Le destin des immigrés (llamativamente poco conocido, incluso entre especialistas) las fecundas posibilidades de los análisis comparativos tanto entre colectivos dentro de un mismo país (noruegos, negros e hispanos en Estados Unidos; Yugoslavos y Turcos en Alemania; Sijs, Pakistaníes y Antillanos en Reino Unido), como de un mismo colectivos en distintos países (estudiando en este caso a magrebíes, o judíos en distintos países de acogida). También Todd ha mostrado cómo para alcanzar su pertinencia dichos análisis deben superar el repertorio cerrado al uso de los estudios sobre integración / aculturación / interculturalidad y para ello se ha concentrado en el análisis de las estructuras familiares y el matrimonio como elemento clave de las estrategias a largo plazo. Pero también nos da un iluminador toque de atención al estudiar otros muchos factores clave rescatados del olvido, como por ejemplo los costos en tiempo, dinero y energía de los mecanismos de segregación de las capas blancas americanas (Todd 1994: 103-105).
} 
este sentido las migraciones internacionales se añaden a los originales demandantes de estudios longitudinales, normalmente pensados en relación con el análisis longitudinal de la educación, la pobreza relativa o los procesos de movilidad social. Sin perjuicio de que esta supuestamente novedosa estrategia metodológica no sucumba a la ilusión biográfica (Bourdieu 1986), de intentar construir unos itinerarios longitudinales sobre un abstracto paisaje euclídeo, sin tener en cuenta que las posiciones y los trayectos no pueden ser cualesquiera.

Un comentario final sobre el impacto de la situación de la estadística de migraciones internacionales en el conjunto de los sistemas estadísticos. No suele tomarse conciencia de que hablar de migrantes internacionales o de población de un país son las dos caras de la misma moneda y que las dificultades de establecer la dimensión de los flujos afectan en la misma medida absoluta (aunque en distinta proporción) también a la medida de los residentes de todas las operaciones estadísticas, y no solamente las demográficas. Esto quiere decir que salvo contextos relativamente desconectados de los flujos migratorios, lo que no es el caso de la mayoría de los países de Europa y Norteamérica, las dificultades de cobertura adecuada o de sesgos significativos en las migraciones internacionales, tienen un elevado impacto en las magnitudes demográficas y económicas de todos los países.

\section{LAS INTERPRETACIONES Y SU SOLUCIÓN CONVENCIONAL}

No abundan de forma explícita los diagnósticos explicativos ${ }^{12}$ de lo que podemos considerar sin duda una anomalía: la pobreza e inconsistencia relativas de las estadísticas de migraciones internacionales. Decir que los sistemas estadísticos nacionales de los distintos países no suelen enfatizar la importancia de estas carencias (destacadas por los especialistas o por algunos informes internacionales) no sería sino ver la otra cara de esta misma irregularidad, con la que normalmente los organismos estadísticos de los países se acostumbran a convivir. Veamos las interpretaciones más usuales para explicar esta mencionada anomalía.

Lo más habitual, lo que podríamos llamar el primer núcleo interpretativo, es destacar las dificultades técnicas de las estadísticas de migración internacional. El repertorio detallado de sus circunstancias puede ser largo: la ausencia de una definición aceptada en la práctica de migrante internacional, la dificultad de hacer un registro de los mismos en la situación actual de enorme movilidad transfronteriza en un mundo cada vez más

\footnotetext{
${ }^{12}$ Las Recomendaciones de Naciones Unidas enuncian categóricamente la necesidad de abordar este asunto: "Understanding the reasons for the lack of comparability is a necessary first step on the path towards enhanced comparability" (United Nations 1998: 15, parágrafo 28), aunque no detalla los resultados de este proceso.
} 
rápidamente interconectado, la ausencia de una tipología estándar de movimientos, la necesidad de coordinar informaciones entre diversos países con diferentes sistemas estadísticos, la diferencia de clasificaciones educativas, económicas y territoriales en origen y destino y algunos otros argumentos, todos ellos de indudable peso.

Sin duda las menciones a las dificultades sustantivas de las estadísticas de migración internacional son claras; sin embargo, no resisten la comparación con otros ámbitos donde estas complejidades técnicas no son menores, pero en los que se han desarrollado enormes esfuerzos por reducirlas. El proceso de unificación conceptual y de definiciones de la Contabilidad Nacional es con mucho más complejo, y actúa en un campo más abierto que las dificultades de medición de un fenómeno como las migraciones, que afecta a personas físicas y cuya tipología más compleja apenas alcanza unas decenas de categorías (United Nations 1998: 19-20, parágrafo 38). Si comparamos esto con los alambicados problemas asociados a la elaboración de las clasificaciones internacionales de actividades económicas, que implican centenares de categorías asociadas a millares de productos, vinculados a distintas unidades de actividad económica que se entrecruzan a distintos niveles (locales, unidades locales, empresas, grupos y conglomerados), lo cierto es que estas llamadas dificultades técnicas parecen un ejercicio escolar elemental. Incluso se puede citar también una excepción de relativo avance fuera de las estadísticas económicas: la elaboración y periódica actualización de la clasificación de enfermedades y causas de muerte y su creciente sofisticación en entramados conceptuales como la codificación múltiple de los distintos procesos patológicos asociados a la defunción.

Ciertamente, incluso en los ámbitos estadísticos más consolidados, los expertos avisan de las limitaciones en la homogeneización internacional de las informaciones y los organismos especializados piden a los países nuevos esfuerzos (mediante mejoras en las operaciones existentes, desarrollo de otras nuevas, integración de fuentes u otras estrategias). Sin embargo, estas mejoras se impulsan a partir de una situación de regular producción de estadísticas estandarizadas, 0 al menos cuyas limitaciones no aparecen evidentes para el usuario no especialista, o mejor, no impiden que los usuarios generales sean capaces de hacer comparaciones regulares de la mayoría de los indicadores clave en el tiempo y en el espacio ${ }^{13}$. Esto es, se dispone de series regulares y razonablemente comparativas de intercambios de bienes y servicios, pero no de flujos de personas, incluso según clasificaciones elementales.

En resumen, es difícil considerar que la anomalía de disponibilidad de información en migraciones internacionales provenga de una especial dificultad técnica del campo de estudio: definición del fenómeno, sujetos a investigar o específicas dificultades de observación de sus características.

Un segundo núcleo de interpretaciones nos hablarían de lo que podemos denominar sesgo temático, de la producción de la estadística oficial en todos los países a partir

\footnotetext{
${ }^{13}$ Véase al respecto el Anejo 1 de este texto.
} 
de la Segunda Guerra Mundial. La orientación general en todos los países, a partir de historias e instituciones muy diferentes, es la gran prioridad otorgada a las estadísticas económicas, medida en recursos de todo tipo aplicados a su producción. Todos los sistemas estadísticos comenzaron por la población y la demografía (durante el siglo XIX), incorporaron las dimensiones sociales en la primera mitad del siglo XX y se volcaron en la producción de estadísticas económicas en su segunda mitad. Con ser esto relativamente claro en términos generales, quizá no dibuja un panorama suficientemente adecuado, puesto que muchas de las operaciones estadísticas tienen diversas facetas y los criterios de clasificación pueden ser muy diversos.

A despecho de enunciar una simpleza, se podría decir que la importancia relativa de cada operación estadística en los sistemas estadísticos entre los años 50 y 90 del pasado siglo es directamente proporcional a su distancia al hogar en el que se concentran la mayoría de los esfuerzos: proporcionar una medida cada vez más precisa del metabolismo económico que mide toda la producción social en términos de flujos monetarios a partir de las convenciones de la Contabilidad Nacional ${ }^{14}$. Si el mercado de trabajo (el paro) puede ser visto como una asunto social (y menos económico), su extraordinaria proximidad a la evaluación del factor trabajo dentro de cada sector económico y en su conjunto determinará que las potentes y complejas encuestas de fuerza de trabajo se hayan impuesto en el medio plazo en todos los países (y con ello se convierten en una fuente preciosa de estadísticas de migraciones ${ }^{15}$ ). Esta explicación sobre minusvaloración temática da cuenta de la peculiaridad de que uno de los aspectos más tratados en relación con las migraciones (las remesas) es precisamente el más conectado con la balanza de pagos de los países.

No obstante, también es cierto que durante la década de los 90 se han consolidado algunos temas u operaciones estadísticas que escapan a esta burda simplificación de suponer que apenas se ha avanzado nada fuera de las estadísticas económicas: las encuestas de uso del tiempo, las estadísticas medioambientales (con toda la ambigüedad y mezcla que acarrean), los estudios de pobreza relativa y las estadísticas sobre diferencias entre mujeres y hombres son algunos de los posibles ejemplos, aunque habrá que ver en qué medida se consolidan estos esfuerzos en el medio plazo.

En tercer lugar, el mayor núcleo de consenso señalaría que la mencionada anomalía se debe a que la migración internacional es un tema políticamente sensible. No es mucha explicación, si además, como veremos, hay muy diversas interpretaciones sobre lo que esto significa.

\footnotetext{
${ }^{14}$ Sobre las limitaciones conceptuales de tal armazón conceptual merece la pena recordar el relevante ensayo de José Manuel Naredo (1987), así como sus esfuerzos prácticos por complementar estas limitaciones en términos de recursos naturales, análisis del patrimonio o ciclos y burbujas inmobiliarias.

${ }^{15}$ Véase al respecto el Módulo especial de 2008 de la LFS o el aprovechamiento la misma fuente a efectos de la estadística de retornos en OCDE (2008: 169).
} 
La mayoría de los informes internacionales y los expertos tan solo aluden al asunto sin muchas precisiones ${ }^{16} \mathrm{y}$ esta generalidad del enunciado no ayuda mucho, ya que la mayoría de las principales informaciones que generan los sistemas estadísticos (como el paro, el crecimiento, el índice de precios, el déficit por cuenta corriente, etc.) son políticamente sensibles. Es más, se le dedican muchos recursos, porque se consideran socialmente relevantes, económicamente necesarios y, por lo tanto, sus resultados es difícil que no se consideren políticamente sensibles. Joaquín Leguina le había dado forma de brillante paráfrasis a esta evidencia al señalar (2002: 139) que un nuevo fantasma recorría Europa en forma de miedo a la inmigración.

Pero en todo caso este núcleo explicativo daría toda la razón a la familia de interpretaciones críticas que ven el fenómeno migratorio como una faceta intrínsecamente asociada a los fenómenos de dominación política (Noiriel 2001), como una fase posterior de los procesos de dominación colonial ${ }^{17}$, o como una imbricación fuerte en los desarrollos de inversión económica y dominación militar (Sassen 1981 y 1988).

Sin embargo, las propias recomendaciones sobre estadísticas de migración internacional de Naciones Unidas dan una pista que puede ser reinterpretada en el contexto de la situación internacional desde los años 1970, cuando al hilo de la crisis económica del shock petrolero de 1973 se inició un ciclo largo de políticas restrictivas de entrada. Se menciona que un distintivo irrenunciable del Estado es el control del acceso de las personas en frontera ${ }^{18}$, incluso en el caso en el que, como a sus nacionales, se les reconozca el derecho de entrada y establecimiento. Esto podría ser reelaborado como sigue.

La dinámica general económica de las últimas décadas (bajo el ambiguo término de globalización) ha supuesto una desregulación sin precedentes de los intercambios de bienes y servicios, así como la instalación de un pensamiento dominante sobre lo

\footnotetext{
${ }_{16}$ "Part of the problem is that international migration is a politically sensitive subject everywhere-from Nigeria and Mexico, which receive migrants respectively from Burkina Faso and Guatemala, to the United States, Europe, and the world's other affluent countries and regions. Political sensitivity is both a cause and result of the data limitations with which analysts and policymakers cope" (Santo Tomás, Summers y Clemens, 2009: v).

17 "Est-il besoin de rappeler que toute émigration est rupture, rupture avec un territoire et par là même avec une population, un ordre social, un ordre économique, un ordre politique, un ordre cultural et moral? Mais, cause de ruptures, l'émigration est elle-même le produit d'une rupture fondamentale: il faut que s'effondrent tous les cadres qui assuraient la cohésion de la société, pour que l'émigration puisse apparaître et se perpétuer. Et on sait que cette rupture initiale est dans le cas de l'émigration algérienne vers la France (et, certainement, dans les cas de beaucoup d'autres émigrations), le produit direct de la colonisation ... Émigrer constitue objectivement (c'est-à-dire à l'insu de tous les partenaires et indépendamment de leur volonté) un acte que, à n'en pas douter, est fondamentalement politique même s'il est dans la nature même du phénomène migratoire, en la forme qu'on lui connaît en France par exemple, de le masquer et de le nier" (Sayad, 1999: 135)

18 "An intrinsic attribute of State sovereignty is the right of the State to determine who can enter and stay in its territory and under what conditions, a right that is tempered in the case of citizens by the right of individuals to leave any country, including their own, and to return to their own country (article 13 of the Universal Declaration of Human Rights)" (United Nations 1998: 8, parágrafo 5),
} 
benéfico de la reducción de la intervención del Estado, salvo un reducido número de tareas asociadas al núcleo duro de la policía administrativa (entre las que figura el control fronterizo). Sin embargo, resulta difícil incrementar el conjunto de flujos e intercambios económicos y al tiempo regular de forma administrativa clásica aquellos intercambios transnacionales en los que interviene la mano de obra, los procesos formativos o las actividades de ocio de las personas. Piénsese en la portentosa dificultad de al tiempo otorgar todo el poder regulador a los agentes privados en la gestión de todos los recursos, pero decidir que uno de ellos (el factor trabajo, pero sólo cuando proviene o se destina al exterior del país) ha de ser regulado con procedimientos administrativos (¿decimonónicos, de planificación centralizada 0 indicativa, de gestión directa...?).

Por lo tanto se podría concluir que, junto a sus indudables dificultades técnicas y su ámbito temático no preferente, quizá el factor clave a la hora de explicar la anómala situación de falta de claro progreso e incluso retrocesos de las estadísticas de migración internacional, podría derivarse de esta contradicción, o de esta difícil, si no imposible, misión. Si ya el establishment internacional lo puede reconocer respecto al cambio climático, las estadísticas de migración internacional no serían sino el incómodo espejo que muestra que en verdad la misión encargada al Estado (controlar las fronteras, inventariando quienes las traspasan en cada momento, desde luego al menos los que no son nacionales) resulta en gran medida fallida, en un contexto de flujos económicos de bienes y capitales extremadamente desregulados.

Adicionalmente esta falta de información puede ser interpretada en un segundo nivel de efectos sociales. Esta relativa ceguera informativa es desde luego funcional a la imagen estereotipada de un proceso de globalización tardoilustrado (para la gente, pero sin personas) inmaterial y puramente tecnológico, en el que no se dispone de informaciones concretas sobre las personas y los grupos implicados, sobre sus costos y sobre los éxitos y fracasos asociados a los mismos. Paradójicamente es esta falta de datos la que adicionalmente no permite contrastar con información empírica visiones grandilocuentes 0 apocalípticas sobre la dimensión de los flujos migratorios (oleadas, invasiones silenciosas, etc.), su impacto en los servicios públicos, las características de las personas implicadas o sus efectos en los países de origen (fuga de cerebros y emprendedores) o destino (sobre la nación).

Por si las anteriores consideraciones parecieran en excesivo abstractas, se han confeccionado las tablas 1 y 2 , en las que se reflejan las disponibilidades de información que cualquier usuario puede consultar en los datos sobre flujos migratorios de Eurostat ${ }^{19}$. En cuanto a un primer nivel de disponibilidad de la información la oferta

\footnotetext{
${ }^{19}$ La información está los ficheros de flujos inmigratorios según territorio de residencia previa (migr_immiprv, http://nui.epp.eurostat.ec.europa.eu/nui/show.do?dataset=migr_immiprv\&lang=en) y flujos de emigración según territorio de destino (mgr_eminxt, http://nui.epp.eurostat.ec.europa.eu/nui/show.do?dataset=migr_ eminxt\&lang=en). La consulta se hizo el 5 de febrero de 2010. Solo se han considerado los frutos intraeu-
} 
Tabla 1.

Número de países para los que hay información de flujos migratorios intra UE por país de origen y sistema estadístico de registro

\begin{tabular}{|c|c|c|c|c|c|c|c|c|c|c|c|c|}
\hline & \multicolumn{5}{|c|}{ Registrado en origen } & \multicolumn{6}{|c|}{ Registrado en destino } & \\
\hline Origen_t & 2002 & 2003 & 2004 & 2005 & 2006 & 2007 & 2002 & 2003 & 2004 & 2005 & 2006 & 2007 \\
\hline at Austria & 26 & 26 & 26 & 26 & 26 & 26 & 16 & 16 & 17 & 17 & 15 & 14 \\
\hline be Belgium & & & & & & & 17 & 17 & 18 & 18 & 16 & 15 \\
\hline bg Bulgaria & & & & & & 26 & 17 & 17 & 18 & 18 & 16 & 14 \\
\hline cy Cyprus & 26 & 26 & 26 & 26 & 26 & 26 & 16 & 15 & 16 & 17 & 15 & 13 \\
\hline cz Czech Republic & 26 & 26 & 26 & 26 & 26 & 26 & 16 & 16 & 17 & 17 & 15 & 14 \\
\hline de Germany (including ex-GDR from 1991) & 26 & & 26 & 26 & 26 & 26 & 16 & 17 & 17 & 17 & 16 & 14 \\
\hline dk Denmark & 25 & 25 & 25 & 25 & 25 & 25 & 16 & 16 & 17 & 17 & 15 & 14 \\
\hline ee Estonia & & & & & & & 17 & 17 & 18 & 18 & 16 & 14 \\
\hline es Spain & 25 & 25 & 25 & 25 & 25 & 25 & 16 & 16 & 17 & 17 & 16 & 14 \\
\hline fi Finland & 25 & 25 & 25 & 25 & 25 & 25 & 16 & 16 & 17 & 17 & 15 & 14 \\
\hline fr France & & & & & & & 17 & 17 & 18 & 18 & 17 & 15 \\
\hline gr Greece & & & & & & & 17 & 17 & 18 & 18 & 16 & 15 \\
\hline hu Hungary & & & & & & & 17 & 17 & 18 & 18 & 16 & 15 \\
\hline ie Ireland & 1 & 1 & 1 & 1 & 1 & 1 & 17 & 17 & 18 & 18 & 16 & 15 \\
\hline it Italy & 25 & 25 & 25 & 25 & & & 16 & 16 & 17 & 17 & 16 & 15 \\
\hline It Lithuania & & 26 & 26 & 26 & 26 & 26 & 17 & 16 & 17 & 17 & 14 & 14 \\
\hline lu Luxembourg (Grand-Duché) & & 25 & 24 & 26 & 26 & 26 & 17 & 16 & 17 & 17 & 16 & 14 \\
\hline Iv Latvia & 26 & 26 & 26 & 26 & 26 & 26 & 16 & 15 & 17 & 17 & 15 & 13 \\
\hline mt Malta & 1 & & & & & & 17 & 16 & 17 & 18 & 15 & 14 \\
\hline nl Netherlands & 26 & 26 & 26 & 26 & 26 & 26 & 16 & 16 & 17 & 17 & 15 & 14 \\
\hline pl Poland & 26 & 26 & 26 & 26 & 26 & 26 & 16 & 16 & 17 & 17 & 16 & 14 \\
\hline pt Portugal & 27 & 27 & & & & & 17 & 17 & 18 & 18 & 15 & 15 \\
\hline ro Romania & 26 & 26 & 26 & 26 & 26 & 26 & 16 & 17 & 18 & 17 & 16 & 15 \\
\hline se Sweden & 26 & 26 & 26 & 26 & 26 & 26 & 16 & 16 & 17 & 17 & 15 & 14 \\
\hline si Slovenia & 26 & 26 & 26 & 26 & 26 & 26 & 16 & 15 & 17 & 17 & 15 & 14 \\
\hline sk Slovakia & 26 & 26 & 26 & 26 & 26 & & 16 & 16 & 17 & 17 & 14 & 15 \\
\hline uk United Kingdom & 26 & 26 & 26 & 26 & 2 & & 17 & 17 & 18 & 18 & 17 & 16 \\
\hline Total general & 441 & 465 & 463 & 465 & 416 & 414 & 444 & 440 & 468 & 469 & 419 & 387 \\
\hline
\end{tabular}

ropeos. En todo caso la consulta a Eurostat ya supone un primer filtro, pues si acudiésemos a las diversas fuentes originales de los países los desajustes serían superiores. 


\section{Tabla 2.}

Número de países para los que hay infomación de flujos migratorios intra UE por país de destino y sistema estadísico de registro

\begin{tabular}{|c|c|c|c|c|c|c|c|c|c|c|c|c|}
\hline & \multicolumn{4}{|c|}{ Registrado en origen } & & & \multicolumn{4}{|c|}{ Registrado en destino } & & \\
\hline Destino_t & 2002 . & 2003 & 2004 & 2005 & 2006 & 2007 & 2002 & 2003 & 2004 & 2005 & 2006 & 2007 \\
\hline at Austria & 16 & 17 & 17 & 17 & 15 & 15 & 26 & 26 & 26 & 26 & 26 & 26 \\
\hline be Belgium & 17 & 18 & 18 & 18 & 16 & 16 & & & & & & \\
\hline bg Bulgaria & 17 & 18 & 18 & 18 & 16 & 15 & & & & & & 26 \\
\hline cy Cyprus & 16 & 16 & 17 & 17 & 15 & 15 & 26 & 26 & 26 & 26 & 26 & 26 \\
\hline Cz Czech Republic & 16 & 17 & 17 & 17 & 15 & 15 & 26 & 26 & 26 & 26 & 26 & 26 \\
\hline de Germany (including ex-GDR from 1991) & 16 & 18 & 17 & 17 & 15 & 15 & 26 & & 26 & 26 & 26 & 26 \\
\hline dk Denmark & 16 & 17 & 17 & 17 & 15 & 15 & 26 & 26 & 26 & 26 & 26 & 26 \\
\hline ee Estonia & 16 & 17 & 17 & 17 & 15 & 15 & & & & & & \\
\hline es Spain & 16 & 17 & 17 & 17 & 16 & 15 & 26 & 26 & 26 & 26 & 26 & 26 \\
\hline fi Finland & 16 & 17 & 17 & 17 & 15 & 15 & 26 & 26 & 26 & 26 & 26 & 26 \\
\hline fr France & 16 & 17 & 17 & 17 & 16 & 15 & & & & & & \\
\hline gr Greece & 17 & 18 & 18 & 18 & 16 & 16 & & & & & & \\
\hline hu Hungary & 16 & 17 & 17 & 17 & 15 & 15 & & & & & & \\
\hline ie Ireland & 17 & 18 & 18 & 18 & 16 & 16 & 1 & 1 & 1 & 1 & 1 & 1 \\
\hline it Italy & 16 & 17 & 17 & 17 & 16 & 16 & 26 & 26 & 26 & 26 & & \\
\hline It Lithuania & 17 & 17 & 16 & 17 & 15 & 15 & & 26 & 26 & 26 & 26 & 26 \\
\hline lu Luxembourg (Grand-Duché) & 17 & 17 & 17 & 17 & 15 & 15 & & 22 & 24 & 26 & 22 & 22 \\
\hline Iv Latvia & 16 & 17 & 17 & 17 & 15 & 15 & 26 & 26 & 26 & 26 & 26 & 26 \\
\hline mt Malta & 16 & 17 & 17 & 17 & 16 & 16 & & & & & & \\
\hline nl Netherlands & 16 & 17 & 17 & 17 & 15 & 15 & 26 & 26 & 26 & 26 & 26 & 26 \\
\hline pl Poland & 16 & 17 & 17 & 17 & 15 & 15 & 26 & 26 & 26 & 26 & 26 & 26 \\
\hline pt Portugal & 17 & 18 & 18 & 18 & 16 & 16 & 27 & 27 & 27 & & 26 & \\
\hline ro Romania & 16 & 17 & 17 & 17 & 15 & 15 & 26 & & & 26 & & \\
\hline se Sweden & 16 & 17 & 17 & 17 & 15 & 15 & 26 & 26 & 26 & 26 & 26 & 26 \\
\hline si Slovenia & 16 & 17 & 16 & 17 & 15 & 15 & 26 & 26 & 26 & 26 & 26 & 26 \\
\hline sk Slovakia & 16 & 17 & 17 & 17 & 15 & 16 & 26 & 26 & 26 & 26 & 26 & \\
\hline uk United Kingdom & 18 & 18 & 18 & 18 & 17 & 17 & 26 & 26 & 26 & 26 & 6 & \\
\hline Total general & 441 & 465 & 463 & 465 & 416 & 414 & 444 & 440 & 468 & 469 & 410 & 387 \\
\hline
\end{tabular}

europea de datos está muy marcada por cinco países para los que no existe en absoluto información de flujos demográficos en el período 2002-2007 considerado (y único disponible): Francia es el más notable, Bélgica el más extraño (ya que es un país con registro de población) y les acompañan en ausencia de datos Grecia, 
Hungría y Malta. Pero a esto hay que añadir que de Bulgaria solo hay datos para 2007, Portugal y Rumanía tienen una situación errática e Irlanda solo reporta las migraciones internacionales con otro país (el Reino Unido). Adicionalmente algunos países tienen enormes demoras en la comunicación como ocurre ahora con Italia, Reino Unido y Eslovakia. El panorama general (gráfico 1) es que sólo se dispone de una situación óptima (flujos registrados tanto en el país de origen como en el de destino) para un tercio de las celdillas de la matriz intraeuropea, para otro tercio disponemos de sólo uno de los puntos de registro y todavía queda entre un $10 \%$ y un $20 \%$ de los casos para los que no existe ninguna información. Tampoco la evolución temporal daba mucho margen al optimismo, antes del nuevo Reglamento que luego se menciona.

Pero las dificultades más sustantivas se refieren a las grandes diferencias en el registro de los flujos, que resalta en el caso de los doblemente registrados, pero que está presente en todos. Si comparamos las diferencias relativas entre la magnitud de los flujos doblemente informados el abanico de situaciones es enorme, tanto dentro de los valores positivos (mayor flujo registrado en el sistema estadístico de destino, como es lógico esperar) como en los valores negativos (que tienen todavía una dispersión mayor). Incluso si hacemos el recuento sólo para aquellos flujos entre países más significativos (por ejemplo, más de 500 personas registradas en origen 0 destino) las diferencias siguen siendo enormes.

Gráfico 1.

Proporción de flujos intraeuropeos para los que existe registro en origen y/o destino

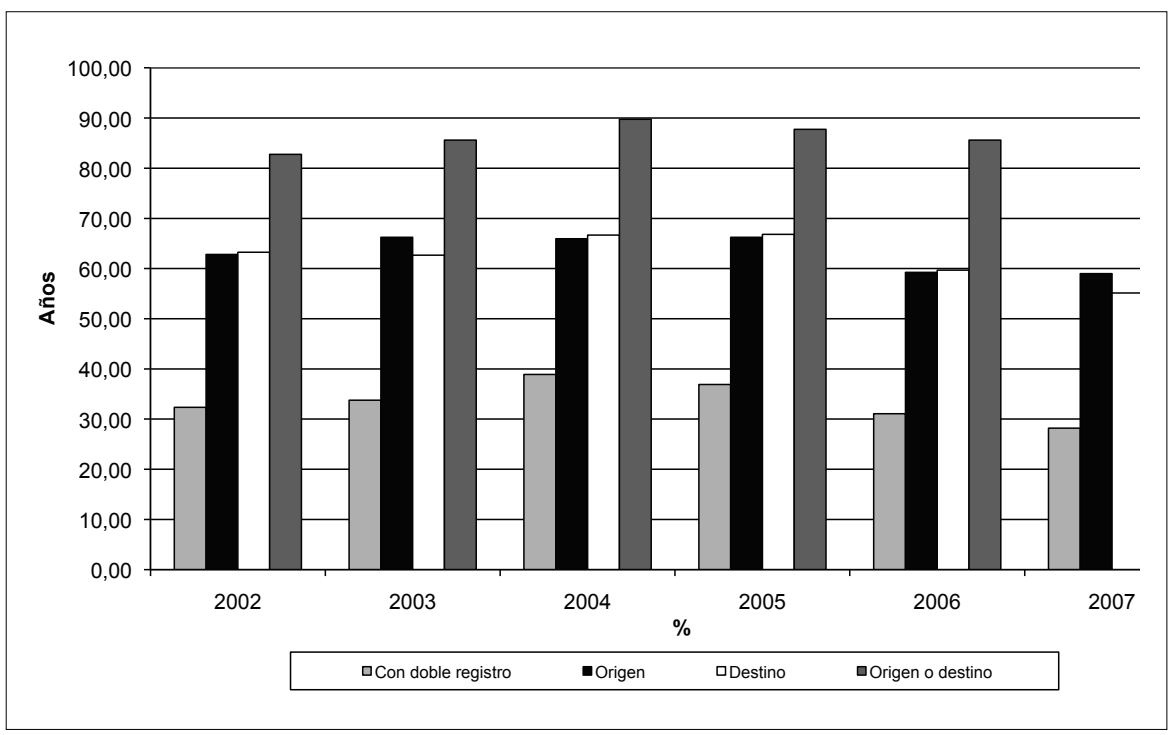


Gráfico 2.

Frecuencias de las diferencias relativas entre países de registro para los mismos flujos migratorios 2002-2007

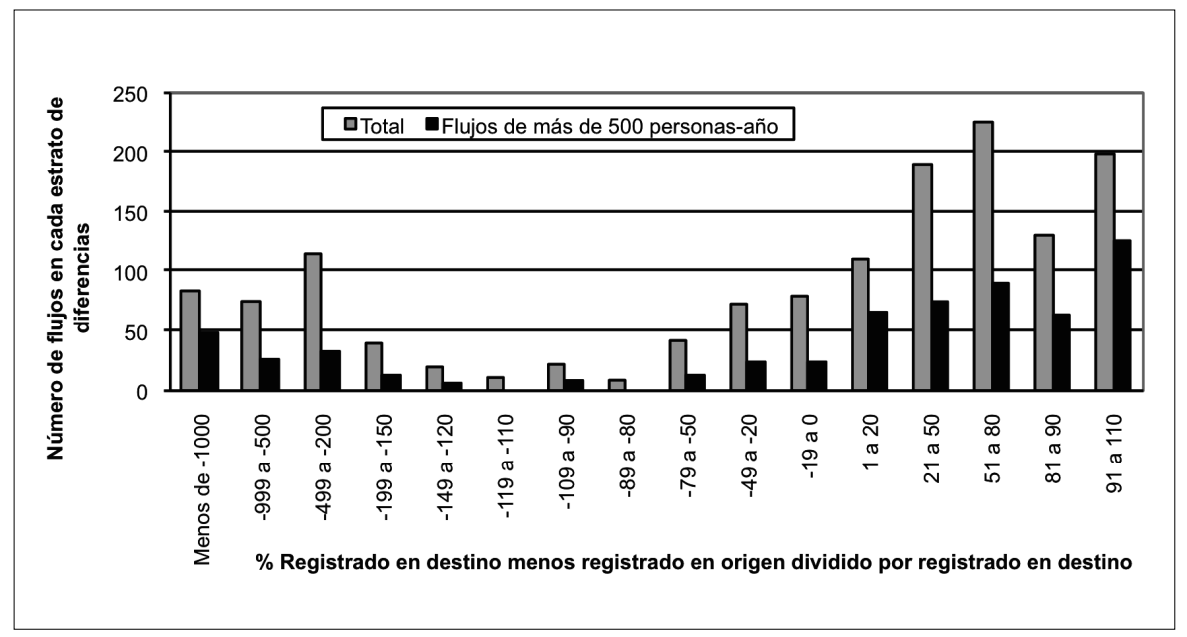

En el fondo de tales dificultades se combinan problemas de cobertura de las fuentes, de diferencias significativas en la definición de migración internacional según países y otros muchos aspectos. Por ello los trabajos que luego se mencionan de estimación de la matriz completa a partir de información parcial y con grandes incoherencias son importantes, pero no hay que olvidar que los ejercicios de estimación son siempre limitados y sus metodologías son muy sensibles a las decisiones de qué conjunto de países se considera que tienen un registro de calidad óptima.

Si además de los totales intentamos obtener información de estructura por edades, características socioeconómicas o motivos de la migración las dificultades informativas se multiplican.

\section{LAS PRINCIPALES INICIATIVAS DE MEJORA EN EL CONTEXTO INTERNACIONAL}

El presente apartado se dedicará precisamente a diseñar el contrapunto del anterior sobre las persistentes dificultades y la anómala situación relativa a las estadísticas de migración internacional. Algo así como una prueba de que incluso en contextos poco favorables existen márgenes para la mejora e iniciativas que consiguen algún éxito a la hora de producir información pertinente.

En el contexto europeo se están desarrollando en los últimos años unos notables esfuerzos por incrementar la mejora de la cobertura, calidad y disponibilidad de esta- 
dísticas de migración internacional, aunque sobre su resultado final todavía es pronto para pronunciarse, habida cuenta de las experiencias de las décadas anteriores. Todos los desarrollos metodológicos sobre la modelización de las migraciones y su integración en los mecanismos de estimaciones y proyecciones de población (Termote 1967; Willekens y Rogers 1978; Courgeau 1980) habían tropezado siempre con la pobreza e incongruencias empíricas de las fuentes de migraciones. Por ello durante la década de los 80 se hicieron diversos esfuerzos para desarrollar un sistema de estimación que aprovechase el carácter matricial de los flujos y también la información disponible sobre las diversas fuentes de captura. Fueron los desarrollos pioneros de Poulain y Watelart (1983), Cagiano de Azevedo (1985) y Kelly (1987).

Los primeros esfuerzos de la Unión Europea se circunscribieron a la recopilación de información sobre trabajadores inmigrantes (Council Regulation (EEC) No 311/76 of 9 February 1976 on the Compilation of Statistics on Foreign Workers) y en todo caso con especificaciones muy generales en cuanto a detalle temático, fuentes, periodicidad y obligatoriedad.

Durante los años 90 Eurostat desarrolló una serie de iniciativas importantes para mejorar la información demográfica y en particular las migraciones: intentando establecer un repertorio armonizado de recopilación de datos (por ejemplo: Eurostat 2000c), integrando dichas informaciones en un sistema que permitiera evaluar la población corriente de la Unión Europea, denominado Now Cast (Eurostat 1999-2001), evaluando las distintas fuentes de información (Eurostat 2000a), comparándolo con los ámbitos migratorios más próximos como Europa del Este y el Mediterráneo (Eurostat $1999 \mathrm{~b}$ y 2000b), investigando la fiabilidad de las fuentes respecto a los movimientos de difícil registro y las migraciones irregulares (Eurostat 1988) e incluso haciendo intentos de capturar de forma empírica los mecanismos causales de las migraciones (Eurostat 2001b).

No obstante, a pesar de la existencia de un proyecto ambicioso durante el principio de los años 90 (Poulain, Debuisson y Eggerickx 1990) y de los enormes desarrollos metodológicos al final de dicha década, parece que el impulso práctico y las realidades de recopilación y mejora no consiguieron fraguar una definitiva mejora de la situación. Esto se debió probablemente en parte a que las sucesivas ampliaciones geográficas de la Unión Europea situaron los desafíos en una dimensión cada vez mayor, quizá también por la desconexión entre consultores y académicos que colaboraban para Eurostat y a la inestabilidad de los recursos técnicos y de acumulación de conocimiento al interior de la propia oficina estadística europea.

Estas dificultades suelen tener un momento especial en el que se hacen claramente patentes y es con ocasión de los trabajos realizados por Eurostat para elaborar unas proyecciones a medio plazo para la población de la Unión Europea. El caso es relevante, ya que regularmente se demanda a Eurostat una proyección demográfica con el fin de basar en la misma los escenarios sobre sostenibilidad económica de cara a los procesos de envejecimiento (European Commission 2006 y 2009). En ese momento la información demográfica en general y las migraciones en particular se sitúan, de una forma 
quizá excesiva, en el corazón del debate del armazón intelectual ${ }^{20}$ del discurso europeo: sostenibilidad, control del déficit, políticas de integración, etc. Se trata, además, de una de las pocas operaciones estadísticas elaboradas directamente por la oficina europea, y no producto de la agregación homogeneizada de datos nacionales. Pero la cuestión crítica no es que el ejercicio proyectivo tenga un mayor o menor grado de acierto respecto a tan solo los primeros años posteriores al mismo (Lanzieri, 2004) sino que en el mismo momento de elaborar el ejercicio de proyección se hace patente que el principal problema es disponer de una serie fiable y homogénea de flujos migratorios recientes, incluso si tan sólo pretendemos disponer de los cuatro o cinco últimos años. Se trata de la misma difícil situación que había dado origen a las metodologías de estimación que intentaban sobrepasar el bloqueo de la inconsistencia de informaciones (Eurostat, 1999a) $)^{21}$.

A pesar de estas dificultades el monumental informe THESIM (Poulain, 2005) hizo una revisión actualizada en catorce capítulos y seis anejos de todos los aspectos en relación con las estadísticas de migraciones, migrantes, permisos de residencia, nacionalizaciones, protección internacional, asilo e intercepción y persecución de las estancias o residencias ilegales. Se trataba no tanto de evaluar una vez más el problema de las dificultades en inconsistencias, sino más bien de evaluar en qué medida un nuevo Reglamento europeo en la materia (esto es, una ley de obligatorio cumplimiento para los Estados miembro) se encontraría con mas o menos dificultades y cual sería la dimensión de los esfuerzos que deberían afrontar los países.

Finalmente el Reglamento entró en vigor en $2007^{22}$ y los países deberían haber empezado a remitir datos en relación de flujos y stocks a partir de los acontecimientos que tienen como fecha de referencia 2008. Solo el tiempo dirá si los ampulosos preám-

\footnotetext{
${ }^{20} \mathrm{No}$ es el lugar para una revisión de estos asuntos, pero conviene mencionar que dichos ejercicios se basan en juego de "compartimentos estancos" en un esquema que aparenta intentar un trabajo interdisciplinar. Primeramente se demanda a los supuestos expertos en los diversos fenómenos demográficos un diagnóstico de la evolución de los principales parámetros (nada menos que a 60 años vista) en ausencia de cambios inesperados o intervenciones políticas. Como no existe ninguna relación entre las migraciones y los fenómenos estrictamente demográficos, la demanda supone una velada invitación a que imaginen lo que les parezca según su juicio experto, independientemente de que las incertidumbres sobre migraciones no pueden apoyarse en ninguna evolución de los factores económicos y sociales que las empujan y modelan. A continuación se apoya en esa estimación puramente demográfica la evolución del comportamiento respecto al mercado de trabajo (tasas de ocupación, jornada laboral) y una estimación de la productividad. Naturalmente frente a lo fácil de esta crítica no es sencillo instrumentar metodologías que recojan la estimación conjunta de los distintos factores explicativos de las migraciones (véase la extraordinariamente completa revisión de Bijak, 2006).

${ }^{21}$ Para disponer de un ejemplo práctico de esta situación consultar las tablas $2 \mathrm{a}, 2 \mathrm{~b}, 2 \mathrm{c}$ y $2 \mathrm{c}$ en cuanto a la situación original de la recopilación de flujos migratorios para el año 1995 (Eurostat, 1999a: 10-13).

${ }^{22}$ Reglamento (CE) No 862/2007 del Parlamento Europeo y del Consejo de 11 de julio de 2007 sobre las estadísticas comunitarias en el ámbito de la migración y la protección internacional y por el que se deroga el Reglamento (CEE) no 311/76 del Consejo relativo a la elaboración de estadísticas de trabajadores extranjeros.
} 
bulos sobre el papel clave de la información sobre migraciones para la Unión Europea consiguen abrir una brecha en el anómalo comportamiento de los sistemas estadísticos respecto a este asunto. En todo caso no sólo se trata de la puesta en marcha de un nuevo esfuerzo con soporte legal de obligatoriedad para los países (un aspecto en el que los expertos habían coincidido que implicaba una gran fragilidad de los mecanismos actuales de recolección internacional de datos ${ }^{23}$ ), sino que además viene acompañado de una serie de iniciativas para la mejora de las fuentes en origen (ILMAS ${ }^{24}$ ) y para la elaboración a un nivel avanzado de estimaciones a partir de datos incompletos 0 no completamente coherentes (MIMOSA ${ }^{25}$ ) de acuerdo al viejo principio de coherencia matricial de los flujos intraeuropeos, con unos desarrollos metodológicos ligeramente actualizados (Raymer y Willekens 2008)

Pero el contexto europeo no es el único hogar de buenas prácticas, limitadas pero relevantes, en relación con las estadísticas de migración internacional. Destaca por su perspicacia y resultados la estrategia de compilación de información sobre migraciones internacionales de la OCDE e intentaremos sintetizar un acercamiento que es totalmente diferente del de Naciones Unidas y Eurostat y que básicamente intenta hacer "de la necesidad virtud". Aunque se reconozca lo adecuado de las definiciones de las recomendaciones de Naciones Unidas, desde el punto de vista práctico sus décadas de dificultoso avance aconsejan (Lemaître 2005), para el ámbito de los países miembros de esta organización, algún atajo. Dos importantes productos ofrece su Departamento de Estadística: a) La Base de Datos sobre Inmigrantes en los países de la OCDE (DIOC y DIOC-Extended ${ }^{26}$, con una información muy detallada a partir de la anterior ronda censal, pero ampliada a la muy relevante información sobre edad y sexo, nivel educativo, profesión, sector de actividad y duración de la estancia de los inmigrantes, con un texto resumen con los principales resultados (OCDE 2008), aunque los datos tienen por referencia los años 2000-2001; y b) La compilación armonizada (Lemaître, Liebig y Thoreau 2006) de estadísticas sobre flujos anuales de inmigrantes internacionales con

\footnotetext{
${ }^{23}$ "It is likely that more countries will be able to provide migrant stock data in the near future. However, owing to the specific mechanisms by which countries collect data on international migrant flows and the United Nations Statistics Division's lack of a clear international mandate with respect to collecting those data directly from the most relevant national authorities, improvements in this area will be limited. It remains unclear whether it is possible to create an international agreement to facilitate the collection of data on migrant flows through national authorities other than national statistical offices. In addition, the issue of how such an agreement would affect the current data-collection process needs further exploration" (United Nations 2004: 216-217).

${ }^{24}$ Implementation of Legislation on Migration and Asylum Statistics, dirigido por Michel Poulain.

${ }^{25}$ Modelling of statistical data on migration and migrant populations a partir de los equipos del NIDI y el CERFM y en relación con el que se disponen ya de resultados de stocks por grupos de edades y sexo, país de nacimiento y nacionalidad y matriz de flujos migratorios (algunos de ellos todavía provisionales): http:/l mimosa.gedap.be/

${ }^{26}$ Publicada en 2008 es un una ampliación de la denominada Base de Datos de Inmigrantes y Expatriados (Dumont y Lemaître, 2005) [consulta 20091230]: http://www.oecd.org/document/51/0,3343, en_2649_33 729_40644339_1_1_1_1,00.html.
} 
autorización de residencia permanente, que normalmente se incorpora a los volúmenes anuales denominados SOPEMI y que intenta al menos disponer de la información más relevante en relación con las políticas migratorias: los datos sobre autorizaciones de trabajo y estudio en el ámbito de los países de la OCDE, y la información sobre el motivo de dicha autorización de estancia, incluyendo en torno al $80 \%$ de los flujos de autorizaciones de residencia permanente en dieciocho países.

$\mathrm{Si}$ a estos dos principales productos añadimos los numerosos trabajos sobre flujos específicos (trabajadores cualificados, estudiantes, ocupados en determinados sectores, resultados educativos de las segundas generaciones, emigración de retorno) la producción de la OCDE es un referente clave especialmente en la relación entre migraciones económicas y políticas orientadas a las mismas.

A pesar de las dificultades la labor de recopilación de Naciones Unidas sigue siendo fundamental en sus dos productos principales: a) La Global Migration Database ${ }^{27}$ ha culminado recientemente un enorme esfuerzo para poner a disposición el gran arsenal de datos de migrantes de las Divisiones de Población y Estadística de NNUU (por nacionalidad o país de nacimiento) a partir de diversas fuentes, aunque lógicamente con un mayor peso de las informaciones censales de todos los países; b) Especialmente interesante y rica es la poco conocida base de datos International Migration Flows to and from Selected Countries ${ }^{28}$, tanto por la longitud temporal de las series, como porque incluye España entre los países seleccionados. Finalmente existe una estimación de Naciones Unidas sobre el stock de inmigrantes que se mantiene actualizada a partir de la información disponible, la última es la revisión de 2008 (United Nations 2009) y las anteriores fueron de 2005 y 2003.

En el ámbito de los microdatos están disponibles diversas iniciativas que permiten el seguimiento de los colectivos de inmigrantes o recientes inmigrantes de acuerdo a distintas estrategias. En relación con ficheros generales, que tienen la ventaja de permitir comparar entre migrantes y no migrantes la recopilación censal promovida por la Universidad de Minnesota (Integrated Public Use Microdata Series-International), permite el acceso a la información censal recopilada en algo más de treinta y cinco países. En el contexto europeo y de otros países es cada vez mas frecuente el uso de las Encuestas de Fuerza de Trabajo, que normalmente incluyen la nacionalidad y las migraciones recientes, lo que abre enormemente el campo para la investigación de los migrantes en relación con el mercado de trabajo y teniendo en cuenta los contextos familiares y de los hogares. Además en 2008 Eurostat incluyó un módulo especial sobre migrantes y descendientes de inmigrantes ${ }^{29}$ (ya disponible en los datos españoles). Dos limitacio-

\footnotetext{
${ }^{27} \mathrm{http}: / /$ esa.un.org/unmigration/ (consultado 30 diciembre 2009).

${ }^{28}$ Lamentablemente no accesible en línea, sino solo a través del CD que acompaña a la publicación y de ciclo de actualización desconocido [consultado 30 diciembre 2009) http://www.un.org/esa/population/publicat ions/2005ittmigflows/2005Rev_International migration flows_Documentation.pdf.

${ }^{29}$ Ya disponible en lo referente a datos españoles (consultado 4 enero 2010). http://www.ine.es/jaxi/
} 
nes plantean dichos datos: el acceso a los microdatos a nivel europeo no es completo y resulta difícil (por las diferentes políticas de confidencialidad en diferentes países) y además existen dificultades metodológicas y prácticas ${ }^{30}$ para la captación de los inmigrantes más recientes que son muy relevantes en el análisis de los datos.

También se han elaborado encuestas ad-hoc o módulos comparativos de migraciones para diversos conjuntos de países, tales como los esfuerzos realizados por la Oficina Internacional de Migraciones, el Banco Mundial y la Organización Internacional del Trabajo, además de las imprescindibles Recomendaciones elaboradas por Bilsborrow, Hugo, Oberai y Zlotnik (1997) ${ }^{31}$. No obstante, estos esfuerzos dibujan un panorama todavía fragmentario.

También hay que mencionar los ambiciosos proyectos de análisis de la migración combinando informaciones a nivel de microdato en origen y destino del proceso migratorio: el Mexican Migration Project (Universidades de Princeton y Guadalajara), el de Migración entre África y Europa (MAFE, INED) y el mencionado push and pull factors (Eurostat 2001b).

Desde el punto de vista de los procesos de asentamiento, incorporación e integración son especialmente pertinentes los ficheros de datos longitudinales, al menos en la medida que en su proceso de renovación de la muestra se hayan cuidado suficientemente los complejos procesos asociados a la captación de los inmigrantes recientes. Simplemente enumerar los más relevantes: LAD-Statistics Canada (Canadá) Longitudinal Administrative Databank (anual desde 1982); SOEP-DIW (Alemania) Längsschnittstudie Sozio-oekonomisches Panel (anual desde 1984); OSA (Países Bajos) Labor Supply Panel (bianual desde 1985); BHPS (Reino Unido) British Household Panel Survey (anual desde 1991); NLSY Bureau of Labor Statistics (Estados Unidos) National Longitudinal Survey of Youth (anual desde 1997); ILFI (Italia) Indagine Longitudinale sulle Famiglie Italiane (bianual 1997-2005).

Se puede por lo tanto observar que a pesar de que las estadísticas de migración internacional dibujan un panorama incompleto y con escasos avances en relación con las demandas de los usuarios, existen en todo caso esfuerzos relevantes que intentan recolectar un panorama lo más general posible, realizar estimaciones en la medida que no existen datos completos 0 armonizados y sobre todo, indagar en las informaciones y las trayectorias de los migrantes y su relación con los no migrantes en origen y destino. Muy lejos de lo que se demanda en relación con el papel clave de las migraciones en nuestro actual contexto, pero en todo caso son prueba que en algunos aspectos o contextos las mejoras son posibles. ¿Se acumulará una masa crítica suficiente como para superar la anomalía mencionada?

menu.do?type=pcaxis\&path=/t22/e308/meto_05/modulo/2008/\&file=pcaxis.

${ }^{30}$ Véase el muy detallado documento metodológico que se elaboró de forma previa a la inclusión del módulo especial sobre Inmigrantes y Descendientes de Inmigrantes (Eurostat, 2005).

${ }^{31}$ Sus capítulos 3 y 4 respecto a los sistemas de recolección de datos de migrantes en general, migraciones laborales y asilo, siguen siendo fundamentales a pesar del tiempo transcurrido. 


\section{LAS ESTADÍSTICAS SOBRE FLUJOS MIGRATORIOS EXTERIORES DE ESPAÑA}

Los notables esfuerzos de compilación (Nicolau 2005) y reconstrucción (Sánchez Albornoz 1988 y 2002; Eiras Roel 1991; Sánchez Alonso 1995; Martínez Shaw 1994; Carmagnani 2004; Babiano y Farré 2002) de las series estadísticas de la migración exterior española todavía dejan algunas lagunas temporales, incluso si sólo pretendemos obtener un montante de flujos absolutos por años durante el último siglo y medio de estadística oficial. Es una ilustración más del conocido fenómeno de que en los países-periodo de emigración la información sobre salidas es sumamente pobre (y la estadística de retornos prácticamente inexistente). En todo caso y a pesar de los diversos esfuerzos contemporáneos (García Fernández 1965) y recientes (Ródenas Calatayud 1994) probablemente el periodo de la migración internacional con origen o destino España entre los años 1955-1975 es el que arroja un punto ciego más relevante en el conocimiento de nuestra demografía de la segunda mitad del siglo XX.

Por fortuna antes del shock inmigratorio el Sistema Estadístico Español había ido construyendo una mejora fundamental de los registros de población de base municipal, intensificando su centralización y coordinación por parte del INE y estableciendo mecanismos más sólidos de comunicación y detección de inconsistencias. Esta mejora de gran calado puesta en marcha a partir de 1996 es lo que se denomina coloquialmente Padrón Continuo. Es un importante paso fundamental para la mejora de la calidad y coherencia de las informaciones y para iniciar el camino a un más intensivo aprovechamiento de las informaciones administrativas, aunque por el tamaño territorial y poblacional del país y la maduración que requiere de las prácticas sociales en relación con la administración se espera todavía mejores resultados en el futuro.

El Padrón Continuo permitió un novedoso y útil planteamiento del Censo de 2001 y sin este instrumento no dispondríamos de una información extraordinaria en cuanto a riqueza, rapidez y detalle territorial sobre los movimientos migratorios. Como tal Registro el Padrón recoge lógicamente con menor cobertura tanto los acontecimientos de residentes en España ocurridos en el exterior, como las emigraciones.

Para mejorar este aspecto, manteniendo el funcionamiento y principios de la reforma de 1996, el INE estableció un sistema de renovación/caducidad de las inscripciones. Se ha aplicado desde diciembre de 2005 a todos los extranjeros exteriores a la Unión Europea que no conste que tienen permiso de residencia permanente y lleven dos años en el país sin materializar algún trámite que implique la constancia de su residencia. El trabajo realizado tiene una enorme importancia en la mejora del sistema (ha implicado en los cuatro primeros años más de tres millones de actualizaciones), así como para el conocimiento de la dinámica migratoria, aunque tiene limitaciones propias de un procedimiento administrativo. El procedimiento no incluyó a los ciudadanos de la Unión Europea, ni tampoco a los ciudadanos de nacionalidad española (aunque al respecto hay algunas iniciativas en marcha), aunque con posterioridad el INE puso en marcha un procedimiento similar (aunque de menor fuerza obligatoria) en relación con los ciudadanos de la Unión Europea. 
Es conocido que en el Padrón de España se pueden inscribir los extranjeros residentes, sin que forzosamente tengan que documentar que cuentan con permiso de residencia en vigor y esta es una característica clave de nuestro sistema legal y de nuestra historia, en la que existe un fuerte papel de la administración local y la que los registros poblacionales son práctica regular desde los siglos XIII-XIV sin interrupción. Es además una excepción en el contexto europeo y lo que hace que la mayoría de los registros de migraciones internacionales basados en los Permisos de Residencia tengan una cobertura inferior a las informaciones españolas basadas en el Padrón.

En todo caso hay que llamar la atención de que la división legal/ilegal, o regular/irregular es una compartimentación demasiado simple de un proceso complejo de caracterizar y sobre todo de captar. Principalmente por la importancia numérica de los colectivos cuya situación legal puede estar en trámite, o no tener perfiles claros, o que desarrollan formas complejas de residencia y migración. Adicionalmente hay que señalar que la ciudadanía de plenos derechos es un conjunto complejo de relaciones con distintos instituciones (y registros) de cada Estado (Hacienda, Seguridad Social, Sanidad, Educación, Policía, Justicia), que en ocasiones tienen lógicas diferentes. En todo caso España se encuentra en una situación ventajosa en relación con el objetivo de acercarse a una medida de mayor calidad de su población residente, ya que resulta más sencillo aproximarse empíricamente a valorar las diferentes situaciones de un colectivo registrado, que evaluar los migrantes efectivamente residentes pero no registrados en los sistemas de Permisos de Residencia.

A pesar del fuerte impulso del Padrón la medida de la población actual que sirve como referente para las operaciones estadísticas (Encuestas a Hogares, Contabilidad Nacional, etc.) sigue descansando por el momento en procedimientos clásicos de estimaciones y proyecciones. A partir del año 2004 el INE desarrollo una metodología de estimación de la población corriente (denominada "Estimaciones de la Población Actual") que es heredera de los procedimientos clásicos, pero que aprovecha la rica información mensual existente y especialmente los datos de migraciones exteriores que facilita el Padrón de Habitantes.

¿Cuál es la encrucijada del sistema estadístico español respecto al desafío de la migración internacional? Por lo visto hasta aquí no habría quizá peor diagnóstico que el que se pudiera hacer desde un ahistoricismo más o menos tecnocrático, que intentase trasplantar variedades botánicas sin percatarse de la particular ecología en que tienen sentido y fruto. Por ello un mínimo diagnóstico de la situación española requeríía de aproximación a su evolución histórica, que es lo que de forma resumida se ha intentado en el esquema 1.

La caracterización genérica de los sistemas estadísticos es siempre parcialmente insatisfactoria, ya que no suele dar cumplida cuenta de todos los matices, tensiones y presupuestos metodológicos desde los que se construyen las informaciones. No obstante, en relación con la población y las migraciones internacionales el Sistema Estadístico Español podría caracterizarse como una situación mixta entre los sistemas clásicos evolucionados y los basados en registros, de acuerdo con el siguiente esquema elemental. 


\section{Esquema 1.}

Caracterización general del Sistema Estadístico Español en relación con la fuentes demográficas en el largo plazo

\begin{tabular}{|c|c|c|c|c|}
\hline $\begin{array}{c}\text { TIPO } \\
\text { DE SISTEMA }\end{array}$ & CONTEXTO & NECESIDADES & INSTRUMENTOS & ESPAÑA \\
\hline \multirow{2}{*}{$\frac{8}{\frac{0}{02}}$} & $\begin{array}{l}\text { Diversos registros fiscales, } \\
\text { de policía, electorales y } \\
\text { administrativos }\end{array}$ & \multirow{2}{*}{$\begin{array}{l}\text { Disponer de } \\
\text { cifras fiables de } \\
\text { población (cada } \\
\text { cierto tiempo). }\end{array}$} & \multirow{2}{*}{$\begin{array}{l}\text { Censos } \\
\text { estadísticos } \\
\text { modernos }\end{array}$} & \multirow{2}{*}{$\begin{array}{c}1768 / 1778 / 1797 \\
\text { y entre } \\
1857-1960\end{array}$} \\
\hline & $\begin{array}{c}\text { Registro regular de hechos } \\
\text { vitales }\end{array}$ & & & \\
\hline \multirow{3}{*}{ 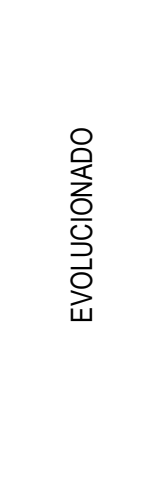 } & $\begin{array}{l}\text { Censos como operación } \\
\text { regular consolidada }\end{array}$ & \multirow{3}{*}{$\begin{array}{c}\text { Disponer de cifras } \\
\text { de población para } \\
\text { la actualidad y el } \\
\text { futuro cercano }\end{array}$} & \multirow{3}{*}{$\begin{array}{c}\text { Censos clásicos } \\
\text { complementados } \\
\text { con trabajos de } \\
\text { estimaciones } \\
\text { intercensales y } \\
\text { proyecciones }\end{array}$} & \multirow{3}{*}{$1981-2001$} \\
\hline & $\begin{array}{l}\text { Desarrollo de grandes } \\
\text { encuestas por muestreo y } \\
\text { Contabilidad Nacional como } \\
\text { eje central del sistema }\end{array}$ & & & \\
\hline & $\begin{array}{c}\text { Desarrollo inicial de diversos } \\
\text { registros poblacionales e } \\
\text { identificadores personales } \\
\text { únicos }\end{array}$ & & & \\
\hline \multirow{3}{*}{ 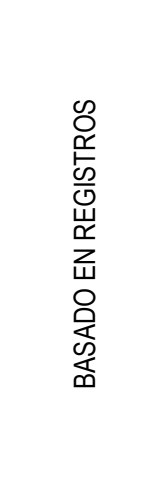 } & $\begin{array}{l}\text { Incremento demandas de } \\
\text { información demográfica } \\
\text { para todo tipo de propósitos }\end{array}$ & \multirow{3}{*}{$\begin{array}{l}\text { Disponer de cifras } \\
\text { de población con } \\
\text { características } \\
\text { básicas y detalle } \\
\text { territorial para la } \\
\text { actualidad y el } \\
\text { futuro cercano }\end{array}$} & \multirow{3}{*}{$\begin{array}{c}\text { Censos y / } \\
\text { o Registros } \\
\text { de Población } \\
\text { complementados } \\
\text { con proyecciones } \\
\text { y estimaciones } \\
\text { temporales, } \\
\text { temáticas y } \\
\text { territoriales }\end{array}$} & \multirow{3}{*}{$\begin{array}{c}2002 \\
\text { en adelante }\end{array}$} \\
\hline & $\begin{array}{l}\text { Integración progresiva de } \\
\text { los diversos registros }\end{array}$ & & & \\
\hline & $\begin{array}{l}\text { Debate sobre la congruencia } \\
\text { de los sistemas y en } \\
\text { relación a los Censos }\end{array}$ & & & \\
\hline
\end{tabular}


El anterior esquema intenta distinguir claramente tres fases en la formación de los sistemas estadísticos y en el papel de las estadísticas demográficas en general y migratorias en particular. La extensión y regularidad de las grandes encuestas por muestreo y las crecientes demandas de integración entre operaciones estadísticas que se iniciaron como separadas ha caracterizado a los sistemas estadísticos después de la segunda guerra mundial. La tercera fase estuvo marcada por el intensivo aprovechamiento estadístico de los registros administrativos en los países nórdicos y en otros de no gran tamaño poblacional y administraciones gestoras muy desarrolladas. Sin embargo, lo que empezó como una característica local ha marcado una tercera etapa para todos los países en la medida que las tecnologías de la información y el tratamiento masivo de datos han puesto al alcance nuevos procesos de integración y aprovechamiento de la información disponible. De este modo lo que empezó como local, empuja también por motivos económicos a un proceso del que no están excluidos ni siquiera los sistemas estadísticos anglosajones, que parecían hasta hace pocos años impermeables a tales desarrollos.

Si los conceptos y ejes vertebradores del anterior esquema está adecuadamente escogidos esto querría decir que el esqueleto fundamental de nuestro sistema estadístico camina hacia una información demográfica de base progresivamente basada en registros y en la integración de los mismos entre sí y con operaciones de tipo muestral, que complementan aquellos aspectos que temporalmente no han sido alcanzados por la integración registral (por ejemplo, todo lo referido a formación y educación en nuestro contexto) o que probablemente nunca estarán adecuadamente tratados en el mismo (por ejemplo lo referido a parejas, convivencia, salud reproductiva, etc.). De la misma forma habría que hacer notar que nadie puede cambiar de contexto (Suecia e Inglaterra no podrían intercambiarse soluciones prêt a porter), pero no es menos cierto que tampoco existen barreras infranqueables.

En los párrafos anteriores se ha mencionado lo relativo a las cifras globales de stocks y flujos migratorios. En este sentido la situación española es bastante sólida en el contexto internacional. Pero la mayoría de estas informaciones básicas solo hacen referencia a los totales y sus características básicas de edad, sexo y país de nacimiento o nacionalidad.

Como se puede observar a la vista de la tabla 3 en este momento en nuestro sistema estadístico la información sobre país de nacimiento o nacionalidad está bastante extendida, pero en general estas informaciones no están complementadas con otras de características socioeconómicas o familiares, a pesar de que tales detalles son extraordinariamente relevantes para la comprensión de los fenómenos migratorios y sus cambios a lo largo del tiempo. No es frecuente que las fuentes españolas recojan (además de los datos sobre lugar de nacimiento y nacionalidad) las migraciones exteriores recientes (salvo el Censo y la EPA) y sin embargo es muy relevante ya que los otros criterios de identificación están mezclando migraciones que pudieron ocurrir a lo largo de casi un siglo anterior a la fecha de referencia.

La información estadística pública ocupa en general huecos que antes han sido transitados por los investigadores o por los organismos especializados y es igualmente el caso de la Encuesta Nacional de Inmigrantes. Es bien conocido que en el caso español 
Tabla 3.

Tratamiento de las variables relacionadas con las migraciones en las operaciones estadísticas del Sistema Estadístico Nacional

\begin{tabular}{|c|c|c|c|c|c|c|c|c|c|}
\hline \multicolumn{10}{|c|}{$\begin{array}{l}\text { OPERACIONES ESTADISTICAS DISPONIBLES SOBRE MIGRACIONES EXTERIORES, POBLACIÓN EXTRANJERA Y POBLACION NACIDA EN EL EXTRANJERO } \\
\text { (actualizado 200909) }\end{array}$} \\
\hline \multicolumn{10}{|c|}{ Sectores o temas del IOE: "Demografia y población", "Trabaio, ingresos y costos selariales" y "Nivel, calidad y condiciones de vidal" } \\
\hline \multirow{2}{*}{$\begin{array}{l}\stackrel{0}{\varepsilon} \\
\stackrel{0}{0}\end{array}$} & \multirow{2}{*}{$\begin{array}{l}0 \\
\frac{0}{0} \\
0 \\
0 \\
0\end{array}$} & \multicolumn{2}{|r|}{ Nombre Operación Estadistica } & \multirow{2}{*}{$\begin{array}{l}\text { Tipode } \\
\text { medida }\end{array}$} & \multirow[b]{2}{*}{ Fuente } & \multicolumn{4}{|c|}{ Variables Principales Difusión } \\
\hline & & $\frac{\pi}{\overline{0}}$ & Denominación & & & \begin{tabular}{|l} 
Nacionalidad \\
/ Nacimiento
\end{tabular} & Personales & socioecnómicas & Migraciones \\
\hline ConVI & 58029 & ECVT & Encuesta de Calidad de Vida en el trabajo & Siock & MTIN & & & & \\
\hline ConVI & 30453 & ECV & Encuesta de Condiciones de Vida & Stock & INE & NCAP & . & - & - \\
\hline ConVI & 30458 & EPF & Encuesta de Presupuestos Familares & Stock & INE & NCASP & & GA & \\
\hline ConVI & 37143 & EFF & Encuesta Financiera de las Familias & Stock & BANESP & * & & & \\
\hline ConVI & 64050 & ENCT & Encuesta Nacional de Condiciones de Trabajo & Stock & MTIN & NCD & $A, S$ & $E, R, 0, R A$ & \\
\hline ConVI & 30450 & TIC-H & Encuesta sobre equipamiento y uso TIC en los hogares & Stock & $\mathbb{N} \mathbb{N E}$ & NCDA & & & \\
\hline Dem & 30243 & CENSO & Censo de Población & Stock & INE & NCD, NMD & $A, S, E C, P$ & $E, R, O, R A$ & $T, R, O R, R T$ \\
\hline Dem & 63051 & CNacE & Concessiones de Nacionalidad Esoañola por Residencia & Flujo & MTIN & NCD & $\mathrm{s}$ & - & MO \\
\hline Dem & 30306 & MNP.D & Defunciones & Flujo & INE & NCD, NMD & A,S,EC & & \\
\hline Dem & 30317 & Efec & Encuesta de Fecundidad & Stock & INE & - & & & \\
\hline Dem & 30311 & & Encuesta de Migraciones & Stock & INE & NCT, RE1A & A,S & $E, R, O, R A$ & $\mathrm{~T}$ \\
\hline Dem & 30319 & ENI & Encuesta Nacional de Inmigrantes & Stock & INE & NMA & A, S, EC, P & $E, R, O, R A$ & $T, R, O R, R T$ \\
\hline Dem & 63007 & EERR & Españoles Resicentes en el Extranjero Retornados & Flujo & MTIN & ESP & 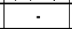 & - & OR,DS \\
\hline Dem & 30259 & ePOBa & Esimaciones de la PoblaciónActual & Stock & $\mathbb{I N E}$ & NCT & A,S & & \\
\hline Dem & 30260 & Padrón & Exolotación Estadistica del Padrön Nunicipal Continuo & Stock & INE & NCD, NMD & A,S & & \\
\hline Dem & 63053 & PermE & Extranieros Autorización de Estancia Estudios en Vigor & Stock & MTIN & NCD & s & - & MO \\
\hline Dem & 63052 & PermR & Extranjeros Autorización de Residencia en Vigor & Stock & MTIN & NCD & A,S & - & MO \\
\hline Dem & 30302 & MNP-M & Matrimonios & \begin{tabular}{|l|} 
Flujo \\
\end{tabular} & INE & NCD & A, S,EC & & \\
\hline Dem & 30304 & MNP.F & Muertes Fetales Terdias & Flujo & INE & NCM, NCP & A,S,EC & & \\
\hline Dem & 30304 & MNP.N & Nacimientos & Flujo & INE & NCM, NCP & A, S, EC & & \\
\hline Dem & 85001 & PERE & Padrón de españoles residentes en el extraniero & Stock & $\mathbb{N}$ NE & ESP & $A, S$ & - & \\
\hline Dem & 30303 & MNPP & Partos & \begin{tabular}{|l|} 
Flujo \\
\end{tabular} & $\mathrm{INE}$ & $\mathrm{NCM}, \mathrm{NCP}$ & A, S,EC & & \\
\hline Dem & 30269 & PPOBC & Proyecciones de Población a Corto Plazo & Stock & INE & $\cdot$ & A, S & & \\
\hline Dem & 30269 & PPOBI & Proyecciones de Población a Largo Plazo & Stock & INE & 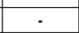 & $A, S$ & & \\
\hline Dem & 68040 & Asilo & Solicitantes de Asilo, Refugio y Protección Intermacional & Flujo & MTIN & NCD & A,S & - & MO \\
\hline Dem & 30271 & Tmort & Tablas de Mortalidad & Tabla & INE & 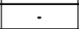 & $A, S$ & & \\
\hline Dem & 30307 & & Variaciones Residenciales & Flujo & INE & NCD, NMD & $A, S$ & & \\
\hline Dem & 63054 & & Visados & Flujo & MTIN & NCD & $A, S$ & - & MO \\
\hline Edu & 42088 & CTNU & Convalidaciones Títulos Extranieros Nivel No Universitario & Fluio & $\mathrm{ME}$ & NCD & $\mathrm{S}$ & $E$ & \\
\hline Edu & 42047 & CTU & Convalidaciones Titulos Extranieros Nivel Universilario & Flujo & ME & NCD & S & $E$ & \\
\hline Edu & 30459 & EADA & Enc. Participación Pobl.Adulta Accividades Aprendizaie & Stock & INE & NCT & $A, S$ & $E, R$ & \\
\hline Edu & 30451 & ETElL & Enc. Transición Eeducativa-Formativa e Inserción Laboral & Stock & INE & NCT & A,S & & \\
\hline Edu & 30405 & EUni & Enseñanzza Universilaria & Flujo & INE & $\cdot$ & $A, S$ & - & \\
\hline Edu & 41001 & EAUni & Enseñanzas Anteriores a la Universidad (Maticula, Graduados, Personal) & Stock & ME & NCT & A,S & & \\
\hline Edu & 30411 & PAU & Pruebas de Acceso a la Universidad & Flujo & INE & . & A,S & $\cdot$ & \\
\hline Prot & 58019 & PAS & Pensiones asistenciales & Stock & MTIN & - & $A, S$ & RE & \\
\hline Prot & 58017 & PCSS & Pensiones contibutivas a la Seguridad Social & Stock & MTIN & - & A,S & RE & \\
\hline Prot & 58021 & Pdes & Prestaciones por desempleo & Flujo & MTIN & $\mathrm{NC}$ & A,S & RE & \\
\hline Prot & 58015 & TASS & Trabajajdores afliliados a la Seguridad Social & Stock & MTIN & NCD & A,S & R,RE & \\
\hline Salud & 54011 & CMBD & Conjunto Minimo Básico de Dátos & Flujo & MSPS & $\cdot$ & & - & - \\
\hline Salud & 30414 & EMH & Encuesta de Morbilidad Hospitalaria & Flujo & INE & - & A,S & - & - \\
\hline Salud & 30455 & ESHS & Encuesta de Salud y Habitos Sexuales & Stock & $\mathbb{N} E$ & - & & & \\
\hline Salud & 30418 & EDAD & Encuesta Discapacidad, Autonomia Personal y Situaciones Dependencia & Stock & INE & $\cdot$ & A,S & & \\
\hline Salud & 54009 & ENS & Encuesta Nacional de Salud & Stock & MSPS & NCT & $A, S$ & & $R, C S$ \\
\hline Salud & 54063 & EDO & Enfermedades de Declaración Obligatoria & Fluio & MSPS & . & . & - & . \\
\hline Salud & 54086 & SIAP & Sistema de Información de Atención Primaria del SNS & \begin{tabular}{|l|} 
Flujo \\
\end{tabular} & MSPS & - & - & - & - \\
\hline Trab & 58002 & PermT & Autorizaciones de Trabajo a Extranieros & Fluio & MEH & NCT & $A, S$ & & \\
\hline Trab & 30188 & EACL & Encuesta Anual de Costete Laboral & Slock & INE & NCD & $A, S$ & $E, R, O, R A$ & \\
\hline Trab & 30133 & EES & Encuesta de Estructura Salarial & Slock & INE & NCD & $A, S$ & $E, R, O, R A$ & \\
\hline Trab & 30308 & EPA & Encuesta de Población Activa & Stock & INE & NCA & $A, S, E C$ & $E, R, O, R A$ & T \\
\hline Trab & 30310 & FL.EPA & Flujos de Poblaciön Activa & Flujo & INE & . & $A, S, E C$ & $E, R, O, R A$ & T \\
\hline Trab & 31062 & SPFT & Mercado de Trabaio y Pensiones en las Fuentes Tributarias & Flujo & MEH & NCT & A, S & & \\
\hline Trab & 59021 & NLR & Movimiento Labora Registrado & Flujo & MEH & NCT & $A, S$ & & \\
\hline & No se difi. & & & & & & & & \\
\hline
\end{tabular}




\begin{tabular}{|l|l|l|l|}
\hline \multicolumn{2}{|l|}{ En relación con la migración, la nacionalidad y el pais de nacimiento } & \multicolumn{2}{|l|}{ En relación con las características básicas } \\
\hline NCT & Nacionalidad Total: Española / Extranjera & A & Año de Nacimiento y/o Edad \\
\hline NCA & Nacionalidad Agrupada: grandes agregaciones de países & S & Sexo \\
\hline NCD & Nacionalidad Detallada: por países & EC & Estado Civil \\
\hline NCM & Nacionalidad de la madre & P & Parentesco \\
\hline NCP & Nacionalidad del padre & En relación con las carácterísticas socioeconómicas \\
\hline NMT & País de Nacimiento Total: España / Extranjero & E & Nivel de estudios \\
\hline NMA & País de Nacimiento Agregado: grandes agregaciones de países & R & Relación con la actividad económica \\
\hline NMD & País de Nacimiento Detallado: por países & O & Ocupación \\
\hline NCM & País de Nacimiento de la madre & RA & Rama de actividad \\
\hline NCP & Pals de Nacimlento del padre & En relaclón con la mlgraclón \\
\hline ESP & Solo para espanoles & I & I Iempo de resıdencla \\
\hline NCASP & Nacionalidad agrupada Sustentador Principal Hogar & LL & Año de llegada \\
\hline RE1A & Residencia en el extranjero hace un año & OR & Lugar de procedencia \\
\hline & & RT & Lugar de residencia hace cierto tiempo \\
\hline
\end{tabular}

el análisis de la inmigración reciente ha sido un terreno privilegiado de los análisis más procedentes de los profesionales de la antropología o de la sociología más vinculada a los análisis concretos (Martínez Veiga 1999; Muñoz Pérez e Izquierdo 1989; Colectivo loé 1997) y hace ocho años ya la producción bibliográfica era difícilmente abarcable (Colectivo loé 2002).

En este contexto es en el que se planteó la necesidad de la Encuesta Nacional de Inmigrantes 2007 como nueva operación estadística que buscase una panorámica general. Desde la perspectiva aquí señalada sus mayores ventajas son precisamente el intento de reconstruir (desde la perspectiva del país de acogida) un panorama completo del proceso migratorio y hacerlo al nivel de los hogares y núcleos de convivencia que se encuentran en el lugar de residencia en el momento de la encuesta.

Otra de sus ventajas es que plantea un panorama sin excluir ningún colectivo de origen o tiempo de llegada y que ha producido resultados en términos poblacionales y no solo muestrales, habiéndose desarrollado una cuidada estrategia que permitiese garantizar la confidencialidad sin apenas limitar el detalle temático, especialmente por países detallados de origen.

\section{Conclusiones}

El panorama de las estadísticas de migración internacional parece ofrecer un paisaje relativamente anómalo en un contexto general de incremento de la información estadística en cuanto a su amplitud, puntualidad o detalle temático y territorial. Tanto los diagnósticos de los expertos como los informes de Naciones Unidas bosquejan un panorama en el que las recomendaciones internacionales de mejora son escasamente atendidas, predominan las fuentes nacionales con conceptos no comparables o simplemente se constata la total ausencia de información, e incluso retrocesos relevantes en la disponibilidad des simples recuentos o información detallada de los migrantes. 
El presente trabajo añade a estos diagnósticos la llamativa constatación sobre los datos disponibles de flujos migratorios entre países de la Unión Europea para los años 2002 a 2007, un periodo y ámbito geográfico particularmente bien nutrido de información en los principales dominios demográficos y sociales. La información recopilada y analizada en el presente texto es muy llamativa tanto en lo referido a la extensión de las omisiones, como a las considerables divergencias en cuanto a las cifras mismas en el caso de aquellos flujos para los que se dispone de la doble información en origen y destino de las migraciones. Este es un aspecto muy relevante en la arquitectura estadística general (incluido las estadísticas económicas), puesto que las dificultades de medición de las migraciones afectan en igual medida absoluta (aunque no relativa) a las cifras de población residente y todas aquellas magnitudes que investigadas de forma muestral requieren de universo poblacional de referencia al que se ajustan sus resultados. Si además se busca información de los migrantes en relación con otras características demográficas y sociales, como se precisa para la elaboración de análisis del impacto social de los flujos y de la efectividad de las medidas de regulación, las disponibilidades actuales resultan todavía más llamativas.

Se ha hecho un repaso a las escasas explicaciones de dicha anomalía y ante la frágil consistencia de las posibles razones debidas a sus complejidades técnicas 0 de captación del fenómeno, queda abierto el campo a aquellas que se plantean la especificidad del objeto de medición estadística: los desplazamientos de personas a través de las fronteras de los Estados. La enorme relación de las migraciones con los conceptos básicos autodescriptivos de cada sociedad y sus identidades (del nosotros en relación con un ellos) y la gran relevancia política de las cifras de población y de migrantes internacionales en un contexto de desregulación globalizada (de mercancías y servicios, pero no de personas) aparecen como dificultades que los sistemas estadísticos apenas pueden enderezar.

No obstante en este contexto se registran un conjunto de proyectos y sistemas de información a nivel internacional que han intentado distintas estrategias para mejorar la situación, que han conseguido algunos resultados notables y a los que los investigadores pueden recurrir en busca de ayuda sobre la información de flujos de migración internacional. En este contexto el esfuerzo de la Unión Europea a partir del nuevo Relamento de Eurostat de 2007 es quizá una de las estrategias mas prometedoras.

El repaso en este contexto de la situación española muestra sus fortalezas y desafíos, así como el papel clave que desempeñan en el sistema tanto nuestro sistema registral basado en el Padrón, como alguno de los esfuerzos complementarios, entre los que la Encuesta Nacional de Inmigrantes 2007 juega un papel novedoso y de aportación original española en el contexto europeo.

La evolución futura de las estadísticas de migración internacional de España depende de cómo evolucione un sistema progresivamente basado en registros en lo que se refiere a sus cifras básicas, solventando el complejo nudo de problemas (que se plantean en todos los países desarrollados) asociados a las diferencias entre cifras registrales y estadísticas. Este aspecto y el resultado de los trabajos que se están desarrollando para 
dar una visión completa del paso de los inmigrantes por nuestros sistemas registrales (Visado, Residencia y Estancia, Padrón, Seguridad Social, Tributos, Registros Civil y Nacionalidad), de forma independiente e integrada, son los que determinarán el grado de esfuerzo de nuestro sistema no solo para suministrar la información demandada por el Reglamento de 2007, sino la manera en que se aprovecha tal obligación para mejorar la riqueza y congruencia de las informaciones de flujos migratorios.

Corresponde ahora al Censo de 2011 y a otras operaciones estadísticas ampliar el panorama facilitado por la ENI-2007, que sin duda ha aportado información relevante (no siempre confirmando los previos lugares comunes del conocimiento no especializado, pero también académico), pero cuya discusión cae fuera de los propósitos de este texto. En todo caso se mantiene el desafío de que el conjunto de operaciones estadísticas terminen desterrando por obsoleto el juicio aquí mencionado sobre la anomalía del conocimiento en materia de migraciones internacionales en España y en Europa.

\section{REFERENCIAS BIBLIOGRÁFICAS}

Anderson, M. J. 1988. The American Census. A social History. New Haven: Yale University Press.

Arribas Macho, J. M. y M. Barbut. 2002. Estadística y Sociedad. Madrid: UNED Ediciones.

Asociación de Historia de da Estadística y de la Probabilidad de España, [AHEPE] 2002. Historia de la Probabilidad y la Estadística I. Madrid: AHEPE.

Asociación de Historia de da Estadística y de la Probabilidad de España, [AHEPE] 2004. Historia de la Probabilidad y la Estadística II. Madrid: AHEPE.

Asociación de Historia de da Estadística y de la Probabilidad de España, [AHEPE] 2006. Historia de la Probabilidad y la Estadística 2006 III. Madrid: AHEPE.

Babiano, J. y S. Farré. 2002. "La emigración española a Europa durante los años sesenta: Francia y Suiza como países de acogida" Historia Social 42: 81-98.

Bijak, J. 2006. Forecasting International Migration: Selected Theories, Models, and Methods, Central European Forum for Migration Research, Working Paper 4/2006.

Bilsborrow, R. E.; Hugo, G. J.; Oberai, A.S. y Zlotnik, H. 1997. International Migration Statistics: Guidelines for Improving Data Collection Systems. Ginebra: International Labour Office.

Bourdieu, P. 1986. "L'illusion biographique." Actes de la Recherche en Sciences Sociales, 62-63: 69-72.

Bras, H. Le. 2000. Naissance de la mortalité. L'origine politique de la statistique et de la Démographie. París: Gallimard / Seuil.

Brian, Er. 2009. Comment tremble la main invisible. Incertitude et marché. París: Springuer. 
Cagiano de Azevedo, R. 1985. "Un metodo per il confronto delle statistiche delle migrazioni internazionali" Noticiario Economico Bresciano 32: 42-49.

Carmagnani, M. 1994. Emigración mediterránea y América. Colombres: Fundación Archivo de Indianos.

Caro Baroja, J. 2004 [1970]. El mito del carácter nacional. Madrid: Caro Raggio.

Colectivo loé 1997. "La inmigración extranjera en Madrid”, OFRIM, suplementos, 17-72. Comunidad de Madrid: Madrid.

Colectivo loé. 2002. Exploración bibliográfica sobre estudios de inmigración extranjera en España. Madrid: Delegación del Gobierno de Extranjería e Inmigración, Ministerio del Interior.

Courgeau, D. 1980. Analyse quantitative des migrations humaines. París: INED.

Courgeau, D. y E. Lelièvre. 2003. "Les motifs individuels et sociaux des migrations", en Démographie: analyse et synthèse. IV Les déterminants de la Migration. París: Institut National d'Études Démographiques.

Desrosières, A. 1993. La politique des grands nombres. Histoire de la raison statistique. Paris: Éditions La Découverte.

Dumont J.C. y G. Lemaître. 2005. Counting Immigrants and Expatriates in OECD Countries: A New Perspective, OECD SOCIAL, EMPLOYMENT AND MIGRATION WORKING PAPERS, DELSA/ ELSA/WD/SEM 4. http://www.oecd.org/dataoecd/34/59/35043046.pdf. Consultado 23 mayo 2011.

Duque, I. 2007. "La observación en directo de la conversión de España en inesperado sueño migratorio de Europa: hipervisibilidad estadística y desafíos explicativos" Revista de Economía Exterior 42: 179-191.

Eiras Roel, A. ed. 1991. La emigración española a ultramar, 1492-1914. Madrid: Tabapress.

European Commission [DG ECFIN] y Economic Policy Committee [AWG] 2006. The impact of ageing on public expenditure: projections for the EU25 Member States on pensions, health care, long term care, education and unemployment transfers (2004-2050), Special Report $n^{\circ} 1 / 2006$ European Economy European Commission Directorate-General for Economic and Financial Affairs.

European Commission [Dg Ecfin] y Economic Policy Committee [AWG] 2009. The 2009 Ageing Report: Economic and budgetary projections for the EU-27 Member States (2008-2060) European Economy, Brussels.

Engels, F. 1999 [1845]. The Condition of the Working Class in England. Oxford: Oxford World's Classics.

Eurostat. 1998. La mesure de la Migration clandestine en Europe Volume 2: rapport des experts, Eurostat Working Papers, 3/1998/E/n07, Catálogo n K5-AP-01-007-FR-I, Luxemburgo, 175 pp.

Eurostat. 1999a. Confrontation des statistiques de Migration intra-européennes: Vers une matrice complète? Eurostat Working Papers 3/1999/E/n0 5, Catálago n KS-AP-01-016-FR-I, Luxemburgo, 35 pp.

Eurostat. 1999b. International Migration Statistics in the Mediterranean Countries Summary report of missions to the 12 project Countries, Eurostat Working Papers 3/1999/E/n¹8. Catálago n ${ }^{0}$ KS-AP01-019-EN-I, Luxemburgo, $51 \mathrm{pp}$. 
Eurostat. 1999-2001. Now-casts on international Migration Part 1: Creation of an information database, Eurostart Working Papers 3/1999/E/n07, Catálago n KS-AP-01-030-EN-I, Luxemburgo, 77pp.

Eurostat. 1999-2001. Now-casts on international Migration Part 1Part II: Searching for the most reliable method, Eurostart Working Papers, 3/2001/E/n 8, Catálago n KS-AP-01-040-EN-I, Luxemburgo, $143 \mathrm{pp}$.

Eurostat. 2000a. Documentation of Eurostat's database on international migration: Acquisition of Citizenship, Eurostart Working Papers 3/2000/E/n³, Catálago n KS-AP-01-022-EN-I, 78 pp. Population by country of birth 3/2000/E/n $n^{\circ}$, Catálago $n^{0} \mathrm{KS}-\mathrm{AP}-01-021-\mathrm{EN}-\mathrm{I}, 28 \mathrm{pp}$.

Eurostat. 2000b, Patterns and trends in international migration in Western Europe, Luxemburgo, 209 pp. ISBN 92-828-9898-9.

Eurostat. 2000c. Statistiques Sociales Européennes. Migration. Luxemburgo, 227pp. ISBN 92-8289217-4.

Eurostat. 2001b. Facteurs d'attraction et de répulsion à l'origine des flux migratoires internationaux, Eurostat Working Papers 3/2000/E/n¹4, Luxemburgo, 184 pp. ISBN 92-828-9899-7.

Eurostat. 2005. Task force Meeting on LFS 2008 ad hoc module on "Labour market situation of migrants and their immediate descendants", Doc. D1/EMPL/08/2005, Luxemburgo.

Eurostat. 2007. Task Force on Core Social Variables. Final Report, Methodological and Working Papers KS-RA-07-006-EN-N. Luxemburgo.

Falkner, R. P. 1895. "The International Statistical Institute." Publications of the American Statistical Association 4: 358-365.

García Fernández, J. 1965. La emigración exterior de España. Barcelona: Ariel.

Gigerenzer, G., Z. Swijtink, T. Porter, L. Daston, J. Beatty y L. KRÜGER. 1989. The Empire of Chance. How Probability Changed Science and Everyday Life. Cambridge: Cambridge University Press.

Ingrao, B. y G. Israel. 1987. La mano invisible. L'equilibrio economico nella historia della scienza. Roma: Laterza.

International Labour Office [ILO] 1922. Recommendation concerning Communication to the International Labour Office of Statistical and Other Information regarding Emigration, Immigration and the Repatriation and Transit of Emigrants, Recommendation R19, adopted November 2, 1922. http:// www.lo.org/ilolex/cgi-lex/convde.pl?R019. Consultada 24 marzo 2011.

Izquierdo, A. 1996. La inmigración inesperada. La población extranjera en España 1991-1996. Madrid: Trotta.

Israel, G. 1996. La mathématisation du réel. Essai sur la modélisation mathématique. París: Seuil.

Kelly, J. 1987. "Improving the Comparability of International Migration Statistics: Contributions of the Conference of European Statisticians from 1971 to Date." International Migration Review 21: 10171037. 
Kraly, E. P. y K. S. Gnanasekaran. 1987. "Efforts to Improve International Migration Statistics: A Historical Perspective." International Migration Review 21: 967-995.

Lanzieri, G. 2004. A Comparison of the Net Migration Data 1995-2002 with the Assumptions in the EU-15 Population Projections, ESTAT/F-1/POP/01(2004)/GL, Working Group on Migration Statistics, Luxemburgo 25-26 February 2004.

Leguina Herrán, J. 2002. "Proyecciones demográficas y de flujos migratorios en España." Procesos migratorios, economía y personas 139-151, en el núm. 1 de la Colección Mediterráneo Económico, Almería: Instituto de Estudios Socioeconómicos de Cajamar.

Lemaître, G. 2005. The Comparability of International Migration Statistics. Problems and Prospects. París: OCDE, Statistics Brief.

Lemaître, G., T. Liebig y C. Thoreau. 2006. Harmonised statistics on immigrant inflows - preliminary results, sources and methods. París: OCDE.

Lévi-Strauss, C. 2001. Race et Histoire. Race et Culture. París: Albin Michel / Éditions UNESCO.

Martínez Shaw, C. 1994. La emigración española a América (1492-1824). Columbres: Fundación Archivo de Indianos.

Martínez Veiga, U. 1999. Pobreza, segregación y exclusión espacial: la vivienda de los inmigrantes extranjeros en España. Barcelona: Icaria.

Moya, J. C. 1998. Cousins and Strangers. Spanish Immigrants in Buenos Aires 1850-1930. Berkeley: University of California Press.

Muñoz Pérez, F., y A. Izquierdo Escribano. 1989. "L'Espagne, pays d'immigration." Population 2: 257-289.

Naredo, J. M. 1987. La economía en evolución. Historia y perspectivas de las categorías básicas del pensamiento económico. Madrid: Siglo XXI.

Nicolau, R. 2005. "Población, salud y actividad". Pp. 77-154 en Albert Carreras and Xavier Tafunell (coord.) Estadísticas históricas de España. Madrid: Fundación BBVA.

Noiriel, G. 2001. État, nation et immigration. París: Folio.

Oecd 2008. International migration Outlook. Annual Repport 2008 Edition. París: SOPEMI.

Poulain, M. y Wattelar Ch. 1983. "Les migrations intra-européennes: à la recherche d'un fil d'Ariane au travers des 21 pays du Conseil de l'Europe": Espace, Populations et Sociétés 1: 11-26.

Poulain, M., Debuisson, M. y Eggerickx, Th. 1990. Projet d'harmonisation des statistiques de migration internationale au sein de la Communauté Européenne, Rapport rédigé à la demande d'Eurostat, Institut de Démographie, Louvain-la-Neuve, 5 vol.

Poulain, M. 2000b. Results of a first survey concerning the implementation of the United Nations recommendations on statistics of international migration, Joint ECE-EUROSTAT-ESCWA work session on Migration Statistics. 
Poulain, Michel [coordinated by] 2005. Towards Harmonised European Statistics on International Migration. Sixth Framework Programme Priority 8.1, Policy-oriented research (SSP), Integrating and Strengthening the European Research Area. Http://www.uclouvain.be/en-7823.html. Consultado 24 marzo 2011.

Porter, T. M. 1995. Trust in numbers. The pursuit of Objectivity in Science and Public Life. Princeton: Princeton University Press.

Raymer, J. y F. Willekens. 2008. International Migration in Europe. Data, Models and Estimates. Nueva York: Wiley.

Ródenas Calatayud, C. 1994. Emigración y economía en España: 1960-1990. Madrid: Cívitas.

Sánchez-Albornoz, N. 1998. Españoles hacia América: la emigración en masa, 1880-1930. Madrid: Alianza Editorial.

Sánchez-Albornoz, N. 2002. "La emigración española a América en medio milenio: pautas sociales." Historia Social 42: 41-57.

Sánchez Alonso, B. 1995. Las causas de la emigración española 1880-1930. Madrid: Alianza Universidad.

Santo Tomas, P. A., L. H. Summers y M. Clemens. 2009. Migrants Count. Five Steps Toward Better Migration Data, Report of the Commission on International Migration Data for Development Research and Policy.

Sasen-Koob, S. 1981. Exporting capital and importing labor: New York City, Center for Latin American and Caribbean Studies. New York University: Occasional Papers, $n^{\circ} 28$.

Sasen-Koob, S. 1988. The Mobility of Labor and capital. A study in international investment and labor flow. Cambridge: Cambridge University Press.

Sayad, A. 1999. "Nationalisme et émigration" en La double absence. Des illusions de l'émigré aux souffrances de l'immigré, 133-159, Seuil (originalmente "Émigration et nationalisme: les cas algérien" en Genèse de l'État moderne en Méditerranée. Approches historique et anthropologique des représentations, Collection de l'École française de Rome, 168, 1993, 407-436).

Skaliotis, M. y D. Thorogoud. 2008. Migration statistics and globalization:challenges for the European Statistical System, 93rd DGINS Conference, 20-21 September 2007. Budapest, Hungary. DGINS 2007/93/II/5.

Stigler, S. M. 1986. The History of Statistics. The Measurement of Uncertainty before 1900. Cambridge, Mass.: Harvard University Press.

Stigler, S. M. 1999. Statistics on the Table. The History of Statistical Concepts and Methods. Cambridge, Mass.: Harvard University Press.

Termote, M. 1967. "Les modèles de migration. Une perspective d'ensemble" Recherches Economiques de Louvain 33: 413-444.

Todd, E. 1994. Le destin des immigrés. Assimilation et ségrégation dans les démocraties occidentales. París: Éditions du Seuil. 
Tooze, J. A. 2001. Statistics and the German State, 1900-1945. The Making of Modern Economic Knowledge. Cambridge: Cambridge University Press.

United Nations. 1980. Recommendations on Statistics of International Migration. Statistical Papers, $\mathrm{n}^{0}$. 58. Sales by Commission Regulation (EEC) No. 761/93 of 24 March No. E.79.XVII.18.

United Nations. 1998. Recommendations on Statistics of International Migration Revision 1, Statistics Division, Statistical Papers Series M, No. 58, Rev. 1 ST/ESA/STAT/SER.M/58/Rev.1, Sales No. E.98.XVII.14. Http://unstats.un.org/unsd/publication/SeriesM/SeriesM_58rev1E.pdf. Consultado 24 marzo 2011.

United Nations. 2004. World Economic and Social Survey 2004: International Migration. New York: ONU. Http://www.un.org/esa/policy/wess/wess2004files/part2web/preface.pdf. Consultada 24 marzo 2011.

United Nations, Department of Economic and Social Affairs, Population Division. 2009. Trends in International Migrant Stock: The 2008 Revision (United Nations database, POP/DB/MIG/Stock/ Rev.2008). http://esa.un.org/migration/index.asp?panel=4. Consultado 24 marzo 2011.

United Nations Economic Commission for Europe (UNECE) / Eurostat. 2008. Guidelines on the use and dissemination of data on international immigration to facilitate their use to improve emigration data of sending countries, Join UNECE/Eurostat Work Session on Migration Statistics Geneva, Switzerland, 3-5 March 2008. http://www.unece.org/stats/documents/ece/ces/ge.10/2008/wp.6.e.pdf. Consultado 24 marzo 2011.

Walter, C. y Brian, E. (dir.). 2007. Critique de la valeur fondamentale. París: Springuer

Willekens, F. y A. Rogers. 1978. Spatial Population Analysis: Methods and Computer Programs, International Institute for Applied Systems Analysis, Laxenburg (Austria). http://www.iiasa.ac.at/Admin/ PUB/Documents/RR-78-018.pdf. Consultado 24 marzo 2011.

IGNACIO DUQUE es Licenciado en Sociología por la Universidad Complutense de Madrid. Ha trabajado principalmente en la producción de estadística pública en el Instituto de Estadística de la Comunidad de Madrid (1985-2000) y desde 2005 en el Instituto Nacional de Estadística, como Subdirector General de Estadísticas de Población e Inmigración y actualmente como responsable de la Unidad de Análisis Sociodemográfico. También ha trabajado como consultor en Informática y Comunicaciones de la Comunidad de Madrid, en la Diputación de Madrid y como consultor independiente.

RECIBIDO: $3 / 11 / 2010$

ACEPTADO: 2/03/2011 